\title{
The genome of a subterrestrial nematode reveals an evolutionary strategy for adaptation to heat
}

Deborah J. Weinstein ${ }^{1}$, Sarah E. Allen ${ }^{1 \uparrow}$, Maggie C. Y. Lau², Mariana Erasmus ${ }^{3}$, Kathryn C.

Asalone $^{1}$, Kathryn Walters-Conte ${ }^{1}$, Gintaras Deikus ${ }^{4}$, Robert Sebra ${ }^{4}$, Gaetan Borgonie ${ }^{5}$, Esta van

Heerden $^{3 \S}$, Tullis C. Onstott ${ }^{2}$, John R. Bracht ${ }^{1 \dagger}$

${ }^{1}$ Biology Department, American University, Washington DC 20016, USA

${ }^{2}$ Department of Geosciences, Princeton University, Princeton, NJ 08544, USA

${ }^{3}$ UFS/TIA Saense Platform, Department of Microbial, Biochemical, and Food Biotechnology, University of the Free State, Bloemfontein 9301, South Africa.

${ }^{4}$ Department of Genetics and Genomic Sciences and Icahn Institute for Genomics and Multiscale Biology, Icahn School of Medicine at Mount Sinai, New York, New York 10029, USA

${ }^{5}$ Extreme Life Isyensya, Gentbrugge, 9050 Belgium.

ๆ Current: Biology Department, Cornell University, Ithaca, NY, 14853, USA

$\S$ Current: North West University, Private Bag X6001, Potchefstroom, 2520, South Africa.

†Contact: John R. Bracht (jbracht@american.edu) 


\section{Abstract}

The nematode Halicephalobus mephisto was originally discovered inhabiting a deep terrestrial aquifer $1.3 \mathrm{~km}$ underground. H. mephisto can thrive under conditions of abiotic stress including heat and minimal oxygen, where it feeds on a community of both chemolithotrophic and heterotrophic prokaryotes in an unusual ecosystem isolated from the surface biosphere. Here we report the comprehensive genome and transcriptome of this organism, identifying a signature of adaptation: an expanded repertoire of 70 kilodalton heat-shock proteins (Hsp70) and avrRpt2 induced gene 1 (AIG1) proteins. We find that positive selection has driven the expansion of Hsp70 genes, which are also transcriptionally induced upon growth under heat stress. We further show that AIG1 may have been acquired by horizontal gene transfer (HGT) from a rhizobial fungus. Over one-third of the genes of H. mephisto are novel, highlighting the divergence of this nematode from other sequenced organisms. This work sheds light on the genomic strategies of adaptation to heat in the first complete subterrestrial eukaryotic genome.

\section{Introduction}

Halicephalobus mephisto was discovered inhabiting a fluid-filled aquifer accessed from the Beatrix Gold Mine in South Africa at $1.3 \mathrm{~km}$ below the surface ${ }^{1}$. Radiocarbon dating indicates the aquifer water is over 6,000 years old ${ }^{1}$, and the lack of surface ${ }^{3} \mathrm{H}$ infiltration, a remnant of atmospheric atomic testing, highlights its isolation from the surface biosphere ${ }^{1}$. The water is warm $\left(37^{\circ} \mathrm{C}\right)$, alkaline ( $\left.\mathrm{pH} 7.9\right)$, hypoxic $\left(0.42-2.3 \mathrm{mg} / \mathrm{L}\right.$ dissolved $\left.\mathrm{O}_{2}\right)$, and rich in biogenic methane $\left(\mathrm{CH}_{4}\right)^{1-3}$. In spite of these challenging conditions, a thriving, complex microbial community exists in this extreme environment including chemolithoautotrophic organisms that extract energy from the subterrestrial rock and fix organic carbon ${ }^{2,4}$. Syntrophic relationships 
link sulphur-oxidizing denitrifying bacteria, sulfate reducers, methanogens and anaerobic methane oxidizing organisms into a complex mutually reinforcing microbial food $w^{2} b^{2}$ that supports a rich assemblage of eukaryotic opportunistic predators including nematodes, rotifers, and protists ${ }^{5}$. With the exception of H. mephisto none of these eukaryotic organisms have been cultured in the laboratory, and none have had their genomes sequenced and analyzed until now.

Nematodes encode small, remarkably dynamic genomes well suited to studies of adaptation $^{6,7}$. Among the most abundant animals on earth, nematodes have adapted to an incredibly diverse set of environments: from hot springs to polar ice, soil, fresh and saltwater ${ }^{8}$, acid seeps ${ }^{9}$, and the deep terrestrial subsurface ${ }^{1}$, with a wealth of comparative genomic data available. Dynamic gene family expansion ${ }^{6,10}$ and shrinkage ${ }^{11}$ have proven good signatures of evolutionary adaptive selection in crown eukaryotes, including nematoda ${ }^{12}$. Here we have performed comprehensive genomic and transcriptomic studies in H. mephisto, giving a first view of the evolutionary adaptive response to a subterrestrial environment, and have identified expanded gene families and patterns of expression under heat stress.

\section{$\underline{\text { Results }}$}

De novo DNA sequence assembly with Illumina data and scaffolding with PacBio reads, yielded a small complete assembly of $61.4 \mathrm{Mb}$ comprising 880 scaffolds with N50 of $313 \mathrm{~kb}$, though $90 \%$ of the sequence is encoded on just 193 scaffolds. The longest scaffold is just under $2.55 \mathrm{Mb}$ (Table 2). Several lines of evidence suggest that this is a highly complete genome. The Core Eukaryotic Genes Mapping Approach (CEGMA) ${ }^{13,14}$ identified 240 of 248 core eukaryotic genes for a completeness of $97 \%$, and tRNscan-SE ${ }^{15}$ identified 352 tRNAs encoding all 20 
amino acids plus selenocysteine. Benchmarking Universal Single Copy Orthologs (BUSCO) ${ }^{16}$ estimated the completeness as $81.4 \%$, but manual inspection of its output shows that 79 “missing” genes are actually detected, with good e-values (median 6e-19). Given that BUSCO's thresholds are established from 8 nematode genes from Clades I, III, and $\mathrm{V},{ }^{17}$ it may not be well suited to divergent Clade IV nematodes such as H. mephisto, since it also scored the P. redivivus genome $\left(98 \%\right.$ complete $\left.^{18}\right)$ as only $82.1 \%$ complete. Therefore considering these divergent matches to be valid, we conclude that for H. mephisto BUSCO detected 946 / 982 orthologous genes, for a completeness of $96 \%$, consistent with CEGMA. Reinforcing the completeness of the H. mephisto assembly and annotation, a quantitative comparison of 3,252 protein domains shows a strong correlation with C. elegans (Figure 1B).

The repetitive component of the $H$. mephisto genome is highly novel. Using a custom RepeatModeler repeat library, RepeatMasker masked $24.3 \%$ of the genome, denoting $21.1 \%$ as interspersed repeats, $87.3 \%$ of which are unknown (Table 1). Evaluation of these sequences with nhmmer ${ }^{19}$ and the DFAM database positively identified $44.3 \%$ of these as helitrons, with $8.8 \%$ retrotransposons and 23.6\% DNA transposons (Table 1). However, consistent with the genomic divergence of $H$. mephisto, this repetitive element repertoire appears extremely different from known elements, including many unique or novel repeat families needing further characterization, and a significant $23.3 \%$ remain unclassified by either algorithm (Table 1 ).

The H. mephisto nuclear genome was annotated with Maker $2^{20}$, TopHat $^{21}$, StringTie $^{22}$, and TransDecoder ${ }^{23}$, and transcripts were clustered with gffcompare to define a total of 16,186 protein-coding loci. An additional 1,023 loci were identified that do not encode proteins over 50 amino acids and are candidate noncoding transcripts, for a total of $n=17,209$ transcribed loci in 
the H. mephisto genome, producing 34,605 transcripts for an average of 2.0 transcripts per locus (Table 2). Intron lengths averaged 473 compared to 320 bp for C. elegans (Table 2).

We used single-copy orthologous proteins (SCOGS) to build a phylogenetic tree placing H. mephisto as a distant relative of free-dwelling Panagrellus redivivus, the nearest fully sequenced nematode relative, within Clade IV (Figure 1A). The comparison of domain counts between $C$. elegans and H. mephisto uncovered two domains strikingly enriched in H. mephisto: avrRpt2-induced gene, AIG1 (84 domains vs. 0) and 70-kilodalton heat-shock protein, Hsp70 (126 domains vs. 15) (Figure 1B). The most over-represented domains in C. elegans are the Meprin And TRAF-Homology (MATH) domain, Fog-2 Homology Domain (FTH) and F-box associated (FBA_2) domains, all apparent lineage-specific expansions in Caenorhabditis ${ }^{24,25}$ (Figure 1B).

We used OrthoVenn ${ }^{26}$ to identify orthologous genes between D. melanogaster, $C$. elegans, $P$. redivivus, and the 16,186 H. mephisto proteins, identifying a set of 5,397 shared among all nematodes, with 3,233 shared among all four invertebrates. Among the $417 \mathrm{H}$. mephisto-specific orthologous groups, the largest cluster was Hsp70, with 107 proteins. The expansion of Hsp70 is particularly evocative because these well-studied heat-activated chaperones re-fold proteins denatured by heat ${ }^{27-30}$ as part of a coordinated response to heatshock $^{31-34}$. Even more intriguing, Hsp70 are expanded in organisms adapted to environmental thermal stress, but not in nematodes parasitic on endothermic hosts, suggesting the expansion of Hsp70 may be a general strategy for adaptation to environmental, not parasitic, heat (Figure 1C). Bayesian phylogenetic analysis of Hsp70 proteins recovered known paralogs specific to cellular compartments including mitochondrial and endoplasmic reticulum (ER) ${ }^{35}$ along with a cluster grouping human, mouse, and nematode genes (Cluster I, Figure 2A). The recovered Hsp70 gene 
tree topology is robust, given that the same structure was recovered by maximum likelihood (Figure S1). The human sequences in Cluster I include well-characterized Hsp70 sequences ${ }^{36}$. Cluster II is a new 37-member H. mephisto-only group, which surprisingly is most closely related to another novel Diploscapter cluster with 59 genes (Cluster III, Figure 2A). These data suggest that the Hsp70 gene family has undergone significant amplification within the Diploscapter and Halicephalobus lineages which, owing to their evolutionary distance (Figure 1A), most likely did not inherit these expanded gene families from a common ancestor. Instead, we propose these genes underwent independent expansions in both lineages under shared evolutionary pressure to adaptat to heat stress: D. pachys is thermotolerant ${ }^{37}$, while $D$. coronatus is a facultative parasite of humans ${ }^{38}$ and a member of the genus has been found in thermal waters $^{39}$.

A signature of adaptive evolutionary change is positive selection, in which mutations altering amino acids $(\mathrm{dN})$ are enriched relative to (presumably neutral) synonymous changes $(\mathrm{dS})$, giving a dN/dS ratio $(\omega)$ greater than $1^{40}$. A value of $\omega$ less than 1 indicates elimination of mutations that alter the amino acid sequence, also known as purifying selection, a hallmark of evolution working to preserve protein function ${ }^{40}$. Given the long branch lengths and potentially selection-driven expansions of Hsp70 genes, we used PAML ${ }^{41}$ to test for positive selection on this gene family. To facilitate this analysis we created a new Bayesian phylogeny using a subset of genes from H. mephisto, the Diploscapter species and C. elegans outgroups, resulting in a well-resolved Bayesian phylogeny (Figure 2B). We ran a branch-sites test in PAML, which estimates two $\omega$ parameters for different codons on a pre-selected "foreground" branch of the phylogenetic tree. The $\omega_{1}$ parameter accounts for pervasive purifying selection at specific sites (codons) but a second $\omega_{2}$ measures values greater or equal to 1 (from neutral to positive 
selection) at other sites. Because $\omega_{2}$ is estimated from the data, it is an indicator of positive selection if it is reported to be above 1 . Furthermore, by performing test twice, once with $\omega_{2}$ fixed at 1 (neutrality, a null model) and once allowing it to be freely estimated from the data, a likelihood ratio test can be used to derive a p-value quantifying the strength of positive selection on a particular branch ${ }^{42}$. In our analysis, positive selection was detected along the long branch leading to the $H$. mephisto cluster: $\omega_{2}$ of 197 on $89 \%$ of amino acids, $\mathrm{p}=0.04$ (Table 3 ), however it was not robust to Bonferroni correction for multiple hypothesis testing, which may be too conservative $^{43}$. Given that this branch has by far the most sites $(89 \%)$ under positive selection, we have made it semi-bold (Figure $2 \mathrm{~B}$ ). Values of $\omega_{2}$ greater than 1 were detected on ten of eleven branches from clusters II and III (Table 3) with four giving a p-value that is significant after correction for multiple hypothesis testing (lines strongly bolded in Figure 2B). In particular, the root of the Diploscapter cluster (Cluster III) showed evidence of strong positive selection (Table 3, Figure 2B). As a control, we tested a Cluster I short branch (L) and mitochondrial gene (M), which showed no evidence of positive selection (Figure 2B, Table 3). Together these data suggest that positive selection has driven the diversification of Hsp70 genes in both Halicephalobus and the Diploscapter lineages.

AIG1 was originally identified as a pathogen response gene in plants ${ }^{44}$, but is also involved in survival of T-cells in mammals, where they were named immune-associated nucleotide binding proteins, (IANs) ${ }^{45}$ or GTPase of immunity-associated proteins (GIMAPs) ${ }^{46}$. These proteins function as GTP-binding molecular switches controlling cell fates ${ }^{46}$. AIG1 was originally reported to be completely absent from nematodes ${ }^{45}$, but by relaxing the statistical stringency we find a single copy of the domain (Y67D2.4) in C. elegans annotated as a homolog of human mitochondrial ribosome associated GTPase 1, MTG1 suggesting a possible divergence 
and expansion of the GTPase superfamily in H. mephisto and other nematodes. Consistent with this, blastp against the nr database and HMMER search of Uniprot reference proteomes identified hundreds of matches with low percent identity $(\sim 30 \%)$ to the AIG1s identified in $H$. mephisto. Nonetheless, the matches range from 250-300 amino acids in length and with e-values from 1e-20 to 1e-30; they come from species as diverse as nematodes, fungi, arthropods, and the parasitic protist Giardia intestinalis. These sequences are generally annotated as uncharacterized or hypothetical proteins, though some are annotated as p-loop containing nucleoside triphosphate hydrolases, suggesting a large GTPase protein family, previously uncharacterized, resides within eukaryotes.

Consistent with the relatively low percent identity of these genes, most did not align well with the $H$. mephisto sequences, and if they did align, they did not show good bootstrap support in phylogenetic trees (data not shown). This suggests the eukaryotic superfamily of GTPases is comprised of distinct and divergent subfamilies, only one of which is the AIG1 domain expanded in H. mephisto. We ultimately were able to obtain good alignments and well-supported phylogenetic trees from only 17 nematode species: an AIG1-like cluster including H. mephisto, Diploscapter coronatus, D. pachys, A. suum, and P. redivivus, while a separate MTG1-related cluster includes the Caenorhabditis sequence and other nematodes (Figure 3A). Notably, the two distinct clusters resolve with 100\% bootstrap support, and while the MTG1-related cluster includes members of all sequenced nematode clades (I,III,IV, and V; no Clade II genomes are currently available ${ }^{47}$ ), the AIG1-like group includes only members of clades III, IV, and V, suggesting a potential origin in the Chromadoria ${ }^{47}$ and extensive amplification in $H$. mephisto (Figure 3A). 
In order to relate these nematode GTPase subfamilies with the previously identified AIG1 / IAN / GIMAP sequences from vertebrates and plants ${ }^{45,46}$ we built a tree including a broader taxonomic sampling beyond nematodes (Figure 3B). To simplify the tree we included only six nematodes in this analysis, five which we previously characterized as having the AIG1-like genes: H. mephisto, P. redivivus, A. suum, D. coronatus, and D. pachys, and the C. elegans representative of the MTG1- related proteins. In this tree the original IAN/GIMAP human-plant cluster was recovered ${ }^{45,48}$ but deeply rooted from the MTG1-related side of the phylogeny with good branch support (Figure 3B). We found two sequences from Rhizophagus irregularis, a plant-root associated fungus, resolve cleanly into the newly discovered AIG1-like group including H. mephisto (Figure 3B). The R. irregularis sequences are from two single-nucleus genome sequences, accessions EXX68593.1 and EXX68414 $4^{49}$. These data suggest that, in contrast to reports of AIG1 being absent in invertebrates ${ }^{45,50}$ Clade III, IV, and V nematodes do have a previously unknown member of this protein family potentially derived from an ancient horizontal gene transfer from a rhizobial fungus. $D$. pachys has been reported to inhabit the rhizobial zone of plant roots ${ }^{37,51}$, where it is ideally situated to acquire horizontally transferred genes from cohabiting fungus. Given that we found five nematode species host orthologs of the AIG1-like domain in their genomes (Figure 3A) the HGT event must have been in a Chromadorean ancestor lineage, not in contemporary D. pachys, though H. mephisto has undergone a spectacular expansion of this gene family that is not present in the other lineages, most of which have only a few copies (Figure 3A). The divergence of suborders Rhabditina $(C$. elegans, D. pachys, D. coronatus) and Tylenchina (P. redivivus and H. mephisto) has been estimated at 22 million years ago using molecular clock methods ${ }^{52}$. A. suum is a member of Spirurina and separated from the Rhabditina 80 million years ago ${ }^{53}$, and the putative HGT event 
must predate these divergences. While inter-domain HGT into eukaryotes has been controversial, transfer from fungus to C. elegans has been documented, setting a precedent for this hypothesized HGT event ${ }^{54}$. Preliminary analysis of codon bias to test for HGT was performed, but caution has been urged given this method's propensity for misleading results ${ }^{55}$; indeed while we found the codon usage of AIG1 genes differed statistically from the rest of $H$. mephisto coding sequences, this was also true for our control datasets including collagens, tubulins, and even Hsp70 (data not shown).

We performed a comprehensive differential gene expression analysis of $H$. mephisto genes whose expression changes under heat stress, identifying 285 heat-upregulated and 675 heat-downregulated transcripts (Figure 4B). Because many upregulated genes were unknown, and the upregulated set is small, Gene Ontology analysis identified no enriched categories. However, the downregulated set of genes was enriched in peptidases, as well as cuticle components and ornithine-oxo-acid transaminase (Table 4 and Figure 4B). The downregulation of cuticular component is entirely driven by 13 collagens (out of 89 genes in the genome).

Hsp70 genes respond to heat (Figure 4A) but with two exceptions they do not make the 2-fold cutoff, and one is actually statistically inhibited on heat stress (Figure 4B). One AIG1 gene is included in the statistically downregulated set (Figure 4B). An intriguing finding is SAX2 (Sensory AXon guidance 2), a protein involved in neuronal development in C. elegans ${ }^{56}$, and whose inactivation causes a variety of developmental, cellular, and behavioral phenotypes ${ }^{57}$. In H. mephisto heat stress causes an isoform switch, with a longer version, MSTRG.2841.1, expressed exclusively at $25^{\circ} \mathrm{C}$ to a shorter version, MSTRG.2841.2, exclusively expressed at 38$40^{\circ} \mathrm{C}$. Thus, sax-2 is identified as a transcript both heat-upregulated (12.9-fold) and heatdownregulated (7.0-fold) on the volcano plot (Figure 4B). The long isoform is 9,286 bp and 
encodes a 3,016 amino acid (aa) protein, with the alternative isoform being 132 basepairs shorter owing to an 84 bp change in transcriptional start site and alternate 3' splice site choice for exon 10 (of 16 exons total). These isoform differences only result in a 16 aa deletion from the predicted protein. Given that the protein is $\sim 3,000$ aa, and the 16 aa change does not significantly alter the encoded domains (identified by HMMER as MOR2_PAG1 N, mid, and C-terminal domains), the functional implications of the transcriptional shift and alternative splicing of SAX2 remains to be investigated in future work.

Among the most strongly heat-induced genes was arginine-rich, mutated in early-stage tumors (ARMET), also called mesencephalic astrocyte derived neurotrophic factor (MANF), a gene involved in the unfolded protein response (UPR) in the endoplasmic reticulum (ER $)^{58}$. ARMET was upregulated over 44-fold by heat in H. mephisto (Figure 4B). In mouse and human cells, ARMET interacts directly with the ER Hsp70 protein BIP / GRP78 ${ }^{59}$. Therefore under hot conditions it may be vital for H. mephisto to co-express Hsp70 and ARMET, particularly if they synergize in responding to the damage due to heat in different cellular compartments, Hsp70 in cytosol and ARMET in ER. It appears that H. mephisto has developed different genomic strategies for upregulating these two genes: mild upregulation of many paralogous genes (Hsp70) versus extremely highly induced expression of a single-copy gene (ARMET). Regardless, ARMET is a strong marker of ER stress with cytoprotective roles ${ }^{58,60,61}$.

Another upregulated gene was Bax Inhibitor-1 (BI-1), which was upregulated 4-fold (Figure 4B). BI-1 is a conserved anti-apoptotic protein that prevents ER stress-induced apoptosis $^{62}$. BI-1 interacts, binds and suppresses IRE1 $\alpha$ activity by cancelling its endoribonuclease and kinase activity activity to promote cell survival ${ }^{63}$. As an ER stress prosurvival factor ${ }^{62}$, BI-1 potentially compensates for the lack of heat induction of AIG1, which 
plays a similar role inhibiting apoptosis ${ }^{46}$, but may be more specifically tuned to the UPR response.

\section{$\underline{\text { Discussion }}$}

As the founding genome sequence of the Halicephalobus genus, H. mephisto is a rich repository of novel biology. H. mephisto separated from Caenorhabditis at least 22 million years ago $^{52,64}$, and likely over 100 million years ago ${ }^{65}$, though calibrating the nematode molecular clock is difficult because of their poor fossil record ${ }^{64}$. Our data suggest that nematodes access the deep subsurface from surface waters facilitated by seismic activity ${ }^{66}$. This transition from surface to deep subsurface would be expected to exert strong selective pressures on their genomes, which in nematodes are particularly evolutionarily dynamic ${ }^{6,7,10-12}$. Therefore we speculate that the H. mephisto divergence may reflect selection more than neutral genetic drift, making it a particularly informative genome. Consistent with this, the expanded Hsp70 and AIG1 gene families are extremely divergent from earlier exemplars, residing on extended branches in phylogenetic analysis (Figures 2 and 3), and positive selection was detected along several lineages of the Hsp70 phylogeny (Figure 2B).

We investigated the novelty of $H$. mephisto's 16,186 protein-coding genes by a combination of domain search (HMMER against the Pfam-A database), blastp against the manually curated high-confidence Uniprot-Swisprot database, blastp against the combined proteomes of 28 nematode species (listed in Methods), and Interproscan 5 analysis. At an e-value of 1e-4 we found 10,567 proteins identified by one or more of these methods, leaving 5,619 unknown genes $(34.7 \%$ of 16,186$)$ lacking domains or any recognizable homology. 
Nevertheless, 3,599 (64.0\%) of the unknown genes are expressed (defined as having at least 5

FPKM across 12 replicates) increasing our confidence they are real genes. Genes whose expression was not detected in our analysis may be expressed at extremely low levels (below 5 FPKM across all replicates) or be expressed under different environmental conditions than the laboratory culture we employed. We conclude that these 5,619 completely unknown genes, and particularly their 3,599 expressed subset, are intriguing candidates for functional adaptation to the deep terrestrial subsurface.

These data are consistent with a previous report that new nematode genomes tend to yield around $33 \%$ proteins that are unrecognizable outside their genus ${ }^{6}$, given that $H$. mephisto is the first of its genus to be sequenced fully. As a control we examined the proteome of Panagrellus redivivus, using the identical protein-identification pipeline, finding $33.8 \%$ unknown genes, quite similar to the numbers for H. mephisto. We also tested the pine wood nematode, Bursaphelenchus xylophilus, and identified 25.6\% unknown genes. Like H. mephisto, P. redivivus and B. xylophilus are the first genomes of their respective genera to be sequenced. When a within-genus comparison is available, the number of novel genes has been reported to drop to around $10 \%{ }^{6}$, which we tested by examining the proteins of Meloidogyne hapla, which has a within-genus match to $M$. incognita in the 28 nematode blast database. Consistent with predictions, we identified $8.4 \%$ unknown proteins in $M$. hapla. Therefore we conclude that while the genomic plasticity we observe for $H$. mephisto is significant, the number of unknown genes is broadly consonant with nematode molecular systematics showing that roundworm genomes are extremely dynamic. The novelty of the $H$. mephisto genes reflect a combination of evolutionary adaptation and a lack of closely related comparative Halicephalobus species in databases. Supporting this, of the 1,730 genes that match nematode genome(s) only, and were 
not identified by Interproscan, Uniprot-Swissprot, or Pfam, 1,480 of them match P. redivivius (Figure S2A), the nearest sequenced nematode relative of $H$. mephisto (Figure 1A). These 1,730 genes are not widely conserved even among roundworms, with only 17 (1\%) of them identified in all 28 species and most (511) identified in only one other species (Figure S2B); unsurprisingly the majority of these $(399,78 \%)$ were only found in P. redivivus.

The assembled genome of $H$. mephisto is smaller $(61.4 \mathrm{Mb})$ than most other sequenced nematode genomes with the exception of M. hapla $(54 \mathrm{Mb})^{67}$ and M. incognita $(47-51 \mathrm{Mb})^{68}$, though similar in size to the most closely related species, P. redivivus $(64.4 \mathrm{Mb})^{18}$. H. mephisto reproduces via parthenogenesis ${ }^{1}$, while both $M$. hapla and $M$. incognita are facultatively parthenogenetic $^{67,68}$. In Caenorhabditis, loss of males has been linked to genome shrinkage ${ }^{11}$. In M. incognita ${ }^{68}$ and $D$. pachys ${ }^{69}$ the loss of sexual reproduction leads to functional haploidization as alleles diverge into paralogs across the genome (leading to most genes being present as duplicate, divergent copies). The conversion of a diploid into a functional haploid genome is associated with three predominant changes: 1) a high degree of heterozygosity as lack of recombination leads to high divergence between alleles, now paralogs, 2) assembly of a haploid genome, and 3) detection of two copies of most genes that are single-copy in other $\operatorname{organisms}{ }^{68,69}$.

The heterozygosity we observed in H. mephisto is modest: The kmer frequency distribution from Illumina reads shows two prominent peaks consistent with approximately $1 \%$ heterozygosity $^{70}$ (Figure S3A) and mapping reads back to the assembly identified 707,190 snps and 55,683 indels (762,873 total variants) confirming an overall snp heterozygosity of $1.15 \%$. In contrast, D. pachys displays $4 \%$ heterozygosity ${ }^{69}$. 
The H. mephisto genome assembly we obtained is largely haplotype-merged: mapping the reads back to the genome shows that $59.7 \mathrm{Mb}$ of the assembled sequence $(97 \%)$ is at haplotype-merged coverage $(102 \mathrm{x})$ and only $1.7 \mathrm{Mb}(3 \%)$ exists as potentially diverged haplotypes at lower coverage (Figure S3B). In agreement, CEGMA analysis of assembled reads (Figure S3C) and fragments (Figure S3D) are predominantly single peaks at approximately 100x coverage.

Allele-to-paralog conversion results in two recognizably distinct gene copies, or paralogs, in the genome. In the D. pachys genome CEGMA found an average of 2.12 copies of each core eukaryotic ortholog ${ }^{69}$ yet in H. mephisto CEGMA ${ }^{13,14}$ reported an average 1.19 copies of each gene while $\mathrm{BUSCO}^{16}$ identified 97\% single-copy genes. We therefore conclude that $H$. mephisto exhibits very little functional haploidization into paralogs, and it may represent an early evolutionary stage in this process.

It is important to verify that amplified Hsp70 and AIG1 gene families do not represent assembly errors. One way this could happen is if an assembler produces multiple overlapping contigs encoding the same locus, leading to artifactually high gene copy numbers. In that case multiple near-identical copies of the genes would be present. To test this we extracted the corresponding nucleotide sequences for each family and performed within-family, all-vs-all blastn at an evalue of 1e-4 and filtered for non-self matches. These nonself matches were relatively divergent: the best nonself within-family blastn match for 112 Hsp70 loci averaged $86.4 \%$ identity at the nucleotide level (Figure S4A) and for 63 AIG1 loci averaged 89.7\% identity (Figure S4B). Given the observed genomic heterozygosity is only $1.15 \%$, these data suggest that the Hsp70 and AIG1 genes are diverged paralogs and neither allelic copies nor redundant misassemblies. As a control we extracted 65 collagen genes and performed the same 
analysis. As might be predicted for an ancient and highly divergent gene family, $74 \%$ of collagen genes lacked within-family nonself blast matches entirely, though those that matched averaged $86.6 \%$, similar to Hsp70 (Figure S4C). These sequence divergences are greater than a control analysis performed by blast of the assembly to itself (Figure S4D). We conclude that the expanded Hsp70 and AIG1 families represent parology rather than assembly redundancy artifact.

Often repetitive elements are collapsed by assemblers, so we checked that Hsp70 and AIG1 are not collapsed repeats, which would cause under-representation of their family diversity. Mapping raw reads onto the assembled genome indicates the collapsed repeats as regions of elevated coverage ${ }^{71}$. By mapping the raw reads back to the $H$. mephisto genome we found that the coverage of both Hsp70 and AIG1 families are not elevated relative to the entire genome (Figure S3E). Overall we conclude that the Hsp70 and AIG1 genes identified in this study are true paralogs, neither over nor under-represented in the genome.

D. pachys is remarkable for having fused its chromosomes together into a single linkage group and eliminating telomeres. However, over 6,000 telomeric repeat-containing reads (at least 4 copies of TTAGGC, the $C$. elegans telomeric repeat ${ }^{72}$ ) were present in the raw Illumina data from H. mephisto. By extracting read pairs with telomeric repeats in at least one read, merging them with $\mathrm{PEAR}^{73}$, and assembling them with MIRA we were able to identify 7 unique subtelomeric regions, suggesting that while the number of $H$. mephisto chromosomes may be reduced relative to $C$. elegans, they are not fused as in D. pachys. Consistent with this, we find two homologs of the telomeric gene Protection of Telomeres (POT1) in H. mephisto relative to the three in C. elegans, all of which are lost in D. pachys ${ }^{69}$. Also in contrast to D. pachys, we were able to identify Telomerase Reverse Transcriptase (TERT) in H. mephisto, suggesting that 
standard telomeres have been retained in the subterrestrial organism and chromosome fusion is not an inevitable consequence of a parthenogenetic lifestyle.

Gene expression provides clues to the adaptation to the warm subterrestrial environment. Among genes whose expression change significantly on exposure to heat, over twice as many are downregulated (675) as upregulated (285) (Figure 4B). We suggest two potential explanations of this phenomenon: the worms may find lower temperatures more stressful given their native conditions are warmer, and therefore activate more genes at $25^{\circ} \mathrm{C}$ relative to $38-40^{\circ} \mathrm{C}$. Or, the downregulation of genes may be itself an adaptation to heat, an idea consistent with the Regulated IRE1 $\alpha$ Dependent Decay (RIDD) pathway of the Unfolded Protein Response $(\mathrm{UPR})^{74,75}$. When the RIDD pathway is activated, degradation of ribosome-bound transcripts is mediated by the endoribonuclease domain of IRE $1 \alpha^{74}$, relieving the immediate protein synthesis demand on the endoplasmic reticulum, and providing existing proteins time to re-fold ${ }^{63,74}$. Our data cannot definitively distinguish these two theories; however, the elevated expression of protein chaperones like Hsp70 under heat exposure (Figure 4A) supports the model in which observed changes in transcriptional profile reflect an adaptive response to higher, rather than lower, temperatures. Combined with the observed heat-induced expression of the anti-apoptotic factor Bax Inhibitor 1 (BI-1) (Figure 4B) this response would help H. mephisto survive the abiotic heat stress of the subterrestrial environment.

While we report significantly expanded Hsp70 and AIG1 families, only the Hsp70 genes are upregulated under heat stress in the laboratory (Figure 4A). Interestingly the worms appear to keep per-gene Hsp70 expression low, even under conditions of heat stress. The per-gene expression of Hsp70 at high temperature is slightly lower than all genes (median Hsp70 FPKM= 2.04, and 2.58 for all genes, Figure 4A), but the dramatic expansion of Hsp70 paralogs 
effectively elevates the gene dosage for Hsp70 to 51-fold higher than a single-copy gene would be. This is a similar order of induction as the ARMET protein, an UPR-related single-copy gene induced 44-fold (Figure 4B), and which may synergize with Hsp70. While many studies have shown induction of Hsp70 genes upon heat-shock, the short-term exposure of non-heat-adapted organism to brief extreme heat ${ }^{31-34}$, organisms under long-term adaptation to heat tend to minimize overexpression of Hsp70 because it has harmful effects on development, fertility, and growth $^{29,76-78}$. Long-term exposure to heat stress led to downregulated Hsp70 expression in both flies $^{79}$ and fish ${ }^{80}$. The subterrestrial environment of H. mephisto is thermostable over time: four readings during a five-year period showed the water temperature was an average of $36.8+/-1.2$ ${ }^{\circ} \mathrm{C}{ }^{2-4,81,82}$. Similarly, we cultured the worms for $2-4$ weeks at constant temperatures $\left(25^{\circ} \mathrm{C}, 38^{\circ} \mathrm{C}\right.$, or $40^{\circ} \mathrm{C}$ ) in the laboratory for RNA isolation and gene expression analysis. Therefore, $H$. mephisto's sustained expression of Hsp70 under conditions of constant stable heat stress implies a functional bypass of the genes' known detrimental effects on growth and development ${ }^{29}$. We hypothesize that the divergent Hsp70 genes in H. mephisto that respond most strongly to elevated temperatures may have been functionally modified to ameliorate these deleterious effects, marking them as important candidates for future study.

We were surprised to find that the expansion of AIG1 in H. mephisto likely mediates abiotic stress aside from heat. These genes respond to abiotic stress, including heat, in Arabidopsis ${ }^{48}$ and in mammals the proteins are involved in immune system function, inhibiting apoptosis during T-cell maturation ${ }^{45}$. Given that $H$. mephisto did not activate AIG1 under heat stress, we posit these genes are involved in responding to hypoxia or other abiotic non-thermal stresses present in the deep terrestrial subsurface. Remarkably, however, heat induced another anti-apoptotic factor in H. mephisto--the Bax Inhibitor $1, \mathrm{BI}-1^{62}$, suggesting a different method 
for blocking apoptotic response under subterrestrial heat stress. Together with our work, these data suggest $H$. mephisto has adapted to the subterrestrial environment by managing unfolded protein stresses while upregulating Hsp70 and inhibiting apoptosis.

We note that the pacific oyster Crassostrea gigas has convergently expanded Hsp70 and AIG1 gene families ${ }^{83}$ and activates the UPR in response to abiotic stress including heat ${ }^{84}$, so $H$. mephisto helps define a general evolutionary adaptive response to heat stress. While the oyster experiences considerable thermal fluctuation, $H$. mephisto does not, as described above. Therefore, the signature of adaptation we report is not limited to cyclical or temporary temperature fluctuations but extends to adaptation to constant warm environments.

The expansion of Hsp70 is shared, also convergently, by distantly related Diploscapter species, soil nematodes which display pronounced thermotolerance ${ }^{37,39}$, and we show here that positive selection has driven Hsp70 expansion in Diploscapter and H. mephisto.These findings may not only relate to environmental heat: $D$. coronatus has been reported as a facultative parasite of humans, so it must survive to $37^{\circ} \mathrm{C}$, human body temperature ${ }^{38}$. Consistent with this, the closest relative of $H$. mephisto is a deadly horse parasite, $H$. gingivalis, which has not been fully sequenced, and has also been reported as a facultative and fatal parasite of humans ${ }^{85}$. Therefore, the genome signature of adapation to heat in H. mephisto, D. coronatus, and D. pachys may serve as a preadaptational bridge to parasitic lifestyles at least in some lineages. Therefore these genomic adaptive strategies are of significant concern to human and animal health, and as our climate warms, it will be increasingly important to understand their evolutionary dynamics. 


\section{Acknowledgements}

The authors acknowledge Laura Landweber, in whose laboratory J.R.B prepared the $H$. mephisto Illumina DNA library. We thank Wei Wang, Jessica Wiggins, and Donna Storton of the Princeton Sequencing Core Facility for assistance with Illumina library preparation and sequencing. We also acknowledge Aaron Goldman for early work done on the H. mephisto project, and Elizabeth Ginsburg for comments on the manuscript. We thank Evgeny Bisk for assistance with the installation and troubleshooting of software on the Zorro Computer Cluster at American University. Mariana Erasmus acknowledges support from the Technology Innovation Agency (TIA), an agency of the Department of Science and Technology (DST) and is grateful to Izelle de Beer and Bernice Jordaan for their assistance in culturing of the nematodes. We are indebted to the logistical support of Sibanye Gold Limited, the management and staff of Beatrix Gold Mine that enabled the capture of $H$. mephisto and follow up samples for metagenomic studies. The work of M.C.Y.L was supported by NSF Grant \#DEB-1441646 to T.C.O, and J.R.B was supported by NIH 1K22CA184297 and a NASA DC Space Grant Consortium grant which funded the RNA and PacBio sequencing.

\section{Author Contributions}

D.J.W., S.E.A. and J.R.B. performed gene prediction and phylgenetic tree construction; D.J.W. and J.R.B. performed transcriptome analysis and wrote the paper, and J.RB. performed genome assembly. M.C.Y.L constructed the multi-locus phylogenetic tree. M.E. and E.v.H. grew the nematodes for RNA. K.C.A. performed analysis of positive selection and K.W.C performed 
repetitive element analysis. G.D. and R.S performed Illumina RNA and PacBio DNA sequencing. G.B. cultured the nematodes for DNA extractions and with T.C.O. provided critical feedback on the manuscript drafts and input into experimental design.

\section{Figure Legends}

Figure 1. Genomic comparison of $H$. mephisto protein-coding genes. A. Multi-loci phylogeny of H. mephisto using 99 single-copy orthologous genes (SCOGS). B. Pfam domain comparison of nonredunant protein domain content in C. elegans versus $H$. mephisto at an e-value cutoff of $1 \mathrm{e}-$ 10. Expanded AIG1 and Hsp70 domain families are marked, along with the MATH, FTH, and FBA_2 domains known C. elegans-specific expansions. C. Examination of Hsp70 family expansion across species, quantifying proteins, not domains, identified using e-values as in B. D. Venn Diagram comparing orthologous gene clusters between D. melanogaster, C. elegans, $P$. redivivus and H. mephisto. Abbreviations: MATH: Meprin And TRAF-Homology domain, FTH: Fog-2 Homology domain, FBA_2: F-box associated domain.

Figure 2. Analysis of Hsp70. (A) Bayesian phylogenetic tree of Hsp70. H. mephisto sequences marked with an asterisk (*) and D. pachys with arrows. Branch numbers indicate posterior probabilities, scale bar represents substitutions per site. (B) Hsp70 protein Bayesian tree used for $\mathrm{dN} / \mathrm{dS}$ analysis of coding sequence. Branch letters A-M correspond to Table 2 for $\omega$ (dN/dS) 
value. Bold branches indicate statistical significance of $\mathrm{dN} / \mathrm{dS}$ p-value after correcting for multiple hypothesis testing, and the long branch is semi-bold because it is statistically significant prior to multiple testing correction with $89 \%$ of sites under positive selection (Table 2). Branch numbers indicate posterior probabilities, scale bar represents substitutions per site. The sequences used in both trees are indicated with Wormbase (nematode) or Genbank Accessions (other species).

Figure 3. Phylgenetic analysis of AIG1. (A) Nematode-only RAxML tree of 17 species showing two clusters: MTG1-related and AIG1-like groups. (B) Nematode, human, Arabidopsis, and fungal RAxML tree illustrating potential HGT event. IAN / GIMAP are synonyms of AIG1, the original names given to plant and vertebrate sequences ${ }^{46}$. Two fungal (Rhizophagus irregularis) AIG1-like sequences highlighted in light red boxes. For both trees, branch numbers indicate bootstrap support from 200 replicates, and scale bar represents substitutions per site. The sequences used in both trees are indicated with Wormbase (nematode), UniProtKB (Rhizophagus irregularis) or Genbank Accessions (other species).

Figure 4. Transcriptome analysis of gene expression in H. mephisto. A. Boxplot showing that Hsp70 genes are induced on heat while AIG1 are unchanged. Box shows median and first and third quartiles, while whiskers indicate the 15 th to 85 th percentiles, and notches represent confidence intervals. P-values obtained by 2-tailed Mann-Whitney test. B. Volcano plot of gene expression fold change under hot (combined replicates: $38^{\circ}, \mathrm{n}=3$, and $40^{\circ}, \mathrm{n}=6$ ) versus $25^{\circ} \mathrm{C}$ control (replicates, $n=3$ ). Statistically altered transcripts, defined as q-value less than 0.05 and 
upregulated or downregulated at least 2 -fold under heat-stress conditions $\left(38-40^{\circ} \mathrm{C}\right)$ relative to $25^{\circ} \mathrm{C}$ controls, are indicated with luminosity of 1 while nonsignificant are luminosity 0.1 . Genes labeled 'Unknown' are novel, as discussed in the text. Proteins encoding no recognizable domain but matching other nematodes by blastp are indicated with the number of nematode species matched (out of the 28 used in constructing the blast database). For genes matching only a single other nematode matches, the species is given in parenthesis. Color key: red: Hsp70, blue: AIG1, green: ARMET, and magenta: peptidases. Abbreviations: Sax-2, Sensory axon guidance 2. LSM, Like SM. BI-1, Bax Inhibitor - 1. ARMET, arginine-rich, mutated in early-stage tumors.

Figure S1. RAxML phylgenetic tree of Hsp70 domains. H. mephisto sequences marked with an asterisk (*) and D. pachys with arrows. Branch numbers indicate bootstrap support, scale bar represents substitutions per site.

Figure S2. A. Analysis of 1,730 H. mephisto genes matching to at least one nematode proteome but otherwise lacking recognizable domains by Interproscan or Pfam, and lacking matches to Uniprot-Swissprot. The evalue threshold was set to 1e-4 and number of matches per species are plotted. B. Histogram showing the number of species matched for each of the 1,730 intranematode proteins.

Figure S3. K-mer frequency and assembly coverage. A. Kmer frequency distribution (23-mers). B. Per scaffold coverage vs percent GC. C. Assembled read coverage from CEGMA. D. Assembled fragment coverage from CEGMA. E. Boxplot of per-base coverage of the $H$. mephisto genome, Hsp70 loci, and AIG1 loci. 
Figure S4. Analysis of degree of similarity at nucleotide level between members of expanded gene families. Self-self blastn was performed with evalue 1e-4, then the percent identity of the first non-self match was plotted. A. Hsp70, B, AIG1, C, Collagen (control), D, full genome (control).

\section{Methods}

\section{H. mephisto culture and isolation of DNA and RNA}

H. mephisto were cultured by standard C. elegans methods ${ }^{86}$, on agar plates seeded with Escherichia coli OP50. For DNA extraction the NucleoSpin kit (Cat \#740952.250, MachereyNagel, Bethlehem, PA, USA) was used. The pellet was resuspended in $540 \mu \mathrm{T} 1 \mathrm{buffer}$ supplemented with $10 \mu \mathrm{l}$ Proteinase K. Lysis was accomplished by 4 cycles of rapid freeze-thaw using a dry ice-ethanol bath, with thawing on a $56^{\circ} \mathrm{C}$ heatblock; cycles were approximately 1 minute per freeze or thaw step. After this the sample was treated with an additional $25 \mu 1$ proteinase $\mathrm{K}$ overnight at $56^{\circ} \mathrm{C}$. After this the manufacturer's protocol was followed for column purification of high-quality DNA, which was verified by gel electrophoresis prior to library construction.

For RNA, the worms were cultured on $5 \%$ agar plates at $25^{\circ} \mathrm{C}, 38^{\circ} \mathrm{C}$, or $40^{\circ} \mathrm{C}$ for $2-4$ weeks prior to harvest, then pelleted in PBS pH 7.7, and flash-frozen or immersed in DNA/RNA Shield Buffer for storage and extraction of nucleic acids. For RNA extraction Zymo's Duet DNA/RNA MiniPrep Plus kit (Cat \# D7003) was used. The worm pellet was resuspended in $300 \mu \mathrm{l}$ of DNA/RNA Shield buffer, transferred to a tube of $0.5 \mathrm{~mm}$ BashingBeads (Zymo Cat \# 
S6002), and homogenized on a vortexer at maximum speed twice for 5 minutes with a 1-2 minute rest period between (for cooling). After this $30 \mu \mathrm{l}$ of PK buffer and $15 \mu 1$ Proteinase K were added, and the solution incubated for 30 minutes at $55^{\circ} \mathrm{C}$, then supplemented with $345 \mu 1$ lysis buffer. After pelleting the insoluble material at $16,000 \mathrm{~g}$ for $1 \mathrm{~min}$, the supernatant was transferred to yellow (for DNA) and green (for RNA) columns as described in manufacturer's protocol for the Duet DNA/RNA MiniPrep Plus Kit, performing in-column DNAse treatment of RNA as recommended.

Based on Agilent BioAnalyzer 2100 output, a total of 12 samples yielded RNA of sufficient quality for sequencing: 3 from $25^{\circ} \mathrm{C}, 3$ from $38^{\circ} \mathrm{C}$, and 6 from $40^{\circ} \mathrm{C}$. For analysis the $38^{\circ} \mathrm{C}$ and $40^{\circ} \mathrm{C}$ samples were considered together as 'high' temperature and the $25^{\circ} \mathrm{C}$ replicates as 'normal' temperature.

\section{Genomic DNA Sequencing}

For Illumina, a TruSeq library with insert size of $387 \mathrm{bp}$ was generated with 9 cycles of PCR after gel purification. This library was paired-end sequenced on a HiSeq2500 with 215 bp reads, yielding 58.7 million pairs. This data was assembled with Platanus ${ }^{70}$ as described in the next section. For PacBio, 30 lanes were run on the RS II system using libraries generated off the same DNA sample used in Illumina.

\section{RNA Sequencing}

Stranded RNA-seq libraries were generated using the KAPA Total Stranded preparation kit (KAPA Biosystems catalog \# KK8484) with an average insert size of $175 \mathrm{bp}$. Samples were 
sequenced on a HiSeq 2500 in High Output mode using 2x50 bp paired-end reads, generating at least $60 \mathrm{M}$ total reads per sample. Preliminary quality control for read mapping was performed using taxMaps version $0.2 .1^{87}$, on a downsampled subset (1\%) with the NCBI BLAST nt and a kmer size of 75 to confirm species etiology for the reads generated.

\section{Genome Assembly}

Raw read kmer analysis was performed with the SoapEC v. 2.01 (from the SOAPdenovo2 package $^{88}$ ), KmerFreq_HA set to kmer 23 and error corrected with Corrector_HA. We found that platanus assembly was optimial at 100x average coverage, so a total of 15,582,039 random paired, error-corrected reads were used in the final assembly. Platanus version 1.2.4 ${ }^{70}$ was used to assemble with a stepsize 2 , kmer of 21, and $-\mathrm{u} 1$. Platanus scaffolding was performed with settings -n 345, -a 386, and -d 62. Subsequently gaps were closed with Platanus gapclose. Scaffolds less than $500 \mathrm{bp}$ were discarded and this assembly was further scaffolded with 30 lanes of PacBio data using the PBJelly component of PBSuite v. 15.8.24. Reapr v. 1.0.18 (perfectmap, -b) was used to break chimeras and erroneous gaps. We removed sequences under $1000 \mathrm{bp}$ and identified 40 bacterial scaffolds by a combination of coverage (less than 26X) and GC content (over 55\%), which blast confirmed as prokaryotic. These sequences were found to encode a complete sphingomonas genome to be described elsewhere, but does not appear to be a deep subterrestrial inhabitant based on metagenomic borehole read mapping. After additional removal of the mitochondrial scaffold, this final $H$. mephisto nuclear genome of 880 scaffolds was used in all further analysis. This assembly has an 
$\mathrm{N} 50$ of $313 \mathrm{~kb}$, with the longest $2.55 \mathrm{Mb}$ and is highly contiguous: the final assembly encodes only 10 gaps encoding 476 bp $(0.0008 \%$ of sequence $)$.

\section{Heterozygosity}

The error corrected reads were mapped back to the final H. mephisto assembly with bwamem v.0.7.12 and mis-mapped reads and PCR duplicates were removed with samtools v. 1.9. Using the remaining 31,867,988 reads, snp variants were called with bcftools v. 1.9 mpileup and then call command with the -mv flag.

\section{Analysis of within-family non-self blastn matches}

Gene family coding sequences were extracted into a fasta file based on the transcript coordinates from the GFF file produced by gene annotation with Maker2 and Stringtie. These fasta files were used to build blast databases. These databases were each queried using blastn (at 1e-4) using the same fasta file as query that was used to build the database (thus, performing allvs-all blastn). The resultant blast output in tabular format (-outfmt 6) was parsed using a custom python script to isolate only the first (best) non-self match from the blast output, and a histogram was generated of the percent identies of these non-self matches. For the genomic assembly allvs-all comparison, the same process was carried out but using the 880 -contig full genome assembly. 


\section{Analysis of coverage}

Genome-wide coverage was calculated using the samtools v. 1.9 depth command on the bamfile generated for heterozygosity analysis, followed by custom parsing of the coverage file with a python script. The same coverage file was parsed using the unique transcript coordinates as described in the "Analysis of within-family non-self blastn matches" section. The per-basepair coverage values for Hsp70, AIG1, and the entire genome were evaluated with custom python script producing a boxplot shown in the figure.

\section{Analysis of Repetitive Sequence}

RepeatModeler $^{89}$ v.1.0.11 was used to create a custom repeat library. This library was screened for accuracy with $\mathrm{HMMER}^{90}$ v $3.1 \mathrm{~b} 2$ to identify mis-classifed protein-coding genes, which were removed. This library was used in a RepeatMasker ${ }^{89} \mathrm{v}$ 4.0.6 run using the default parameters. The initial RepeatMasker run designated $21.07 \%$ of the genome as consisting of transposable elements, of which $18.48 \%$ of the total genome, or $87.7 \%$ of the repeat segments, as unclassified repeats. Subsequently, nhmmer ${ }^{19}$ analysis was run on the identified repeats using the DFAM database ${ }^{91}$ with e-value set to $1 \mathrm{e}^{-2}$ to accommodate the highly divergent genome.

\section{Gene discovery}

Maker2 ${ }^{20}$ version 2.38.1 was utilized to run Augustus and SNAP as ab initio predictors to make comprehensive gene predictions for H. mephisto, and incorporating 28 nematode proteomes as hints along with the RNA-seq data. These gene predictions were refined utilizing Tophat2- StringTie-Ballgown suites of programs ${ }^{21,22}$, which also estimate expression levels. Tophat2 v.2.1.1 was used to align the RNA-seq data against the H. mephisto genome with 
Maker2 predicted genes as a input .gff3 file. The resulting .bam files were fed into StringTie v. 1.3.4, to generate a transcriptome annotation of each, as well as quantify the expression levels and estimate the abundance of each transcript, which were subsequently unified using Stringtie's merge function ${ }^{22}$. Ballgown v. 2.12.0 plots the gene abundance and expression data for visualization, from the StringTie output data ${ }^{22}$.

Together StringTie and Maker2 predicted 34,605 transcripts across 12 different RNAseq datasets, which map to a distinct set of 17,209 unique loci as defined by gffcompare. From these loci the longest protein sequence predicted by $\operatorname{TransDecoder}^{23}$ v. 5.3.0, was used in domain comparisons with C. elegans, the reference proteome UP000001940_6239 (19,922 nonredundant proteins) from Ensemb1 RELEASE 2018_04. TransDecoder was run in strict mode, requiring at least 50 amino acids, and only the single best cds prediction per transcript retained, to obtain the nonredundant set of 16,186 protein-coding genes.

The 28 nematode proteomes used are as follows (all obtained from WormBase Parasite, https://parasite.wormbase.org):

ancylostoma_ceylanicum.PRJNA231479.WBPS12.protein.fa ascaris_suum.PRJNA62057.WBPS12.protein.fa brugia_malayi.PRJNA10729.WBPS12.protein.fa bursaphelenchus_xylophilus.PRJEA64437.WBPS12.protein.fa caenorhabditis_angaria.PRJNA51225.WBPS12.protein.fa caenorhabditis_brenneri.PRJNA20035.WBPS12.protein.fa caenorhabditis_briggsae.PRJNA10731.WBPS12.protein.fa caenorhabditis_elegans.PRJNA13758.WBPS12.protein.fa caenorhabditis_japonica.PRJNA12591.WBPS12.protein.fa 
caenorhabditis_nigoni.PRJNA384657.WBPS12.protein.fa

caenorhabditis_remanei.PRJNA248909.WBPS12.protein.fa

caenorhabditis_sinica.PRJNA194557.WBPS12.protein.fa

caenorhabditis_tropicalis.PRJNA53597.WBPS12.protein.fa

diploscapter_coronatus.PRJDB3143.WBPS12.protein.fa

diploscapter_pachys.PRJNA280107.WBPS12.protein.fa

dirofilaria_immitis.PRJEB1797.WBPS12.protein.fa

haemonchus_contortus.PRJEB506.WBPS12.protein.fa

heterorhabditis_bacteriophora.PRJNA13977.WBPS12.protein.fa

loa_loa.PRJNA246086.WBPS12.protein.fa

meloidogyne_hapla.PRJNA29083.WBPS12.protein.fa

meloidogyne_incognita.PRJEB8714.WBPS12.protein.fa

necator_americanus.PRJNA72135.WBPS12.protein.fa

onchocerca_volvulus.PRJEB513.WBPS12.protein.fa

panagrellus_redivivus.PRJNA186477.WBPS12.protein.fa

pristionchus_exspectatus.PRJEB6009.WBPS12.protein.fa

strongyloides_ratti.PRJEB125.WBPS12.protein.fa

trichinella_spiralis.PRJNA12603.WBPS12.protein.fa

trichuris_suis.PRJNA179528.WBPS12.protein.fa

\section{Gene Expression}

After gene discovery, expression analysis was carried out using Ballgown ${ }^{92}$ v. 2.12.0

following the protocol as described ${ }^{22}$. Genes with less than 5 total reads across all 12 replicates 
were filtered from the analysis. Replicates were grouped into 'high' $\left(38-40^{\circ} \mathrm{C}\right)$ or 'low' $\left(25^{\circ} \mathrm{C}\right)$ and the Ballgown stattest() function used to identify those genes statistically different between high and low temperatures. The output of stattest() include q-value and fold-change and these were used to generate the volcano plot as a scatterplot with ggplot2 in R. Significantly heat-regulated genes were defined as exhibiting a q-value less than 0.05 and upregulated or downregulated at least 2-fold under heat-stress conditions $\left(38-40^{\circ} \mathrm{C}\right)$ relative to $25^{\circ} \mathrm{C}$ controls. For the boxplots of gene expression the FPKM values at high or low temperature were exported as a text file and imported into Python 2.7.14 where a custom script was used to construct the boxplots with matplotlib 2.2.2.

\section{Analysis of unknown genes}

H. mephisto genes were analyzed by blastp (evalue 1e-4) against a collection of 28 nematodes as outlined for Gene Discovery. When blasting with controls P. redivivus, M. hapla, or B. xylophilus we created a separate database removing itself (to avoid the trivial selfmatching) and replaced it with the $H$. mephisto proteome, keeping a 28 nematode species comparison set. For all analyses we also performed blastp against the uniprot-swissprot manually curated database (1e-4), Hmmer domain search against the PfamA database (1e-4), and Interproscan 5.30-69.0 running TIGRFAM 15.0, Hamap 2018_03, SMART 7.1, PRINTS 42.0, and Pfam 31.0. Custom python scripts were used to combine output of all analyses and identify true unknown genes.

\section{Venn Diagram}


The genome of each species was uploaded onto the selected template on the website OrthoVenn (http://www.bioinfogenome.net/OrthoVenn/).

\section{Domain Comparisons}

$\mathrm{HMMER}^{93}$ was used to identify protein domains from both $H$. mephisto and C. elegans nonredundant protein predictions with evalue 1e-10. Domain counts of $H$. mephisto were compared to $C$. elegans using a custom python script.

\section{Multi-locus phylogenetic tree}

Phylogenetic relatedness of Halicephalobus mephisto relative to other nematode species in Clade IV was determined by constructing a maximum likelihood tree of single-copy orthologous genes. Protein-coding sequences of 21 nematodes were downloaded from WormBase (release WBPS9) on March 26, 2018. They are Bursaphelenchus xylophilus, Ditylenchus destructor, Globodera pallida, Globodera rostochiensis, Meloidogyne floridensis, Meloidogyne hapla, Panagrellus redivivus, Parastrongyloides trichosuri, Rhabditophanes sp. KR3021, Steinernema carpocapsae, S. feltiae, S. glaseri, S. monticolum, S. scapterisci,

Strongyloides papillosus, S. ratti, S. stercoralis, and S. venezuelensis. The three outgroups were Clade V nematode proteomes, namely Diploscapter pachys PF1309 (NCBI project number PRJNA280107), Heterorhabditis bacteriophora (NCBI project number PRJNA438576), and Caenorhabditis elegans (WormBase release WS264). Following the procedures of OrthoMCL $\mathrm{v} 2.0 .9^{94,95}$, the proteome files were modified to the required format by OrthoMCL (step5), and then filtered to remove sequences that are shorter than 10 amino acid residues and have less than 
$20 \%$ of stop codons (step6). An All-verse-All BLAST ${ }^{96}$ search amongst all proteomes was performed as suggested by OrthoMCL step7, which involved creating a BLAST-searchable protein database with masking information, followed by a BLASTp search with an e-value threshold of 1e-5 (“-evalue 1e-5"), and results were stored in a tab-delimited file ("-outfmt 6"). The BLASTp results were parsed using orthAgogue v1.0.3 $3^{97}$, which filtered out protein pairs with overlap less than 50\% (-o 50) and BLAST (or bit) scores below 50 (-u) before identifying valid protein pairs. the single-copy orthologous groups shared by the 22 proteomes. This step substituted OrthoMCL step8 to step11. The resultant orthologs.abc file was then used for clustering (inflation index, $-\mathrm{I}=2.0$ ) and creating orthologous groups (OrthoMCL step 12-13). Orthologous groups that contained a single protein sequence from each of the 22 genomes were considered as single-copy orthologous groups (SCOGs). Sequences of 99 identified SCOGs were aligned individually using MUSCLE v3.8.31 ${ }^{98}$ and the default parameters, and trimmed using trim $\mathrm{Al}^{99}$ to remove residual positions that were shared by less than $50 \%$ of the sequences in the multiple sequence alignments. Trimmed alignments were manually examined to make sure there was no spurious sequences or poorly aligned regions, and each was evaluated by ProtTest v3.4.2 $2^{100}$ to identify for the best substitution model. Sequences of these 99 SCOGs were concatenated by taxa. Using this final multiple sequence alignment of 43,188 amino acid positions (including 34,549 distinct patterns) and the specific best substitution model identified for individual SCOGs, partitioned phylogenetic analysis was performed using raxml-ng v0.4.1b to find the best maximum-likelihood tree. The substitution models used were JTT $+\mathrm{G}, \mathrm{JTT}+\mathrm{G}+\mathrm{F}$, $\mathrm{JTT}+\mathrm{I}+\mathrm{G}, \mathrm{LG}+\mathrm{G}, \mathrm{LG}+\mathrm{G}+\mathrm{F}, \mathrm{LG}+\mathrm{I}+\mathrm{G}, \mathrm{LG}+\mathrm{I}+\mathrm{G}+\mathrm{F}, \mathrm{RtREV}+\mathrm{I}+\mathrm{G}+\mathrm{F}, \mathrm{VT}+\mathrm{I}+\mathrm{G}, \mathrm{WAG}+\mathrm{I}+\mathrm{G}$, and $\mathrm{WAG}+\mathrm{I}+\mathrm{G}+\mathrm{F}$. Robustness of tree topology was evaluated by 100 iterations of bootstrap analysis. 


\section{Go Analysis}

The find_enrichment.py script from the GOATOOLS v0.6.10 package ${ }^{101}$ was used under default settings to examine the 285 upregulated and 675 downregulated genes relative to the entire set of proteins. GO terms were assigned using Interproscan 5.30.69 ${ }^{102}$.

\section{Hsp70 and AIG1 Tree Building}

For Hsp70 full-length proteins were aligned, and for AIG1 the proteins were broken into domains using the envelope coordinates provided by HMMER and a custom Python script. We labeled the domains by order within the original protein. Alignments performed with MAFFT ${ }^{103}$ v.7.017 and refined manually to minimize indels. Trees were generated with MrBayes ${ }^{104}$ v.3.2.6, using the blosum rate matrix and invgamma rate variation; and RAxML 8.2.12 ${ }^{105}$ with the PROTCATBLOSUM62 rate matrix and 200 bootstrap replicates. All non-mephisto sequences in Figure 2 are identified using their Wormbase ParaSite (https://parasite.wormbase.org/index.html) gene identifier. (For a list of species downloaded from Wormbase, see Gene Discovery section of Methods). For Figure 3 we prepended non-mephisto sequences with the shortest possible (normally two letter) genus and species abbreviation (e.g., 'Dp' for Diploscapter pachys) followed by ${ }^{\prime \wedge}$ prior to the Wormbase ParaSite gene identitifer. Non-nematode gene sequences are indicated by their NCBI accession numbers and UniProtKB identifier for Rhizophagus irregularis.

\section{PAML with branch-sites model}


Using Codeml (PAML v.4.9) ${ }^{41,106}$ branch site $\omega$ was estimated using runmode $=0$, seqtype $=1$, model $=2$, NSsites $=2$, in the Codeml control file. Each branch was estimated twice: once with a neutral model (fix_omega $=1$ and omega $=1$ ) and once using a purifying selection model (fix_omega $=0$, omega $=1$ ). The $\mathrm{p}$ values were determined using the likelihood ratio test (LRT) statistic $2 \Delta l$ compared against $\chi^{2}$ with critical values of $3.84,5 \%$ significance level, and 6.63 , for $1 \%$ significance ${ }^{107}$.

\section{Analysis of subtelomeric sequences}

Extraction of telomere repeat-containing read pairs (at least one of which contains at least 4 copies of TTAGGC) from raw Illumina data was performed using a custom Python script. These read pairs were merged at their overlaps using PEAR $^{73}$ v0.9.8, and the resultant fused reads were assembled with MIRA $^{108}$ v4.0. The resulting contigs were manually inspected and redundant sequences were collapsed to obtain an estimate of subtelomeric region number.

\section{Data Availability}

This Whole Genome Shotgun project has been deposited at DDBJ/ENA/GenBank under the accession SWDT00000000. The version described in this paper is version SWDT01000000.

The raw Illumina DNA and RNA data, and PacBio DNA data are available on the Sequence Reads Archive (SRA) at accession PRJNA528747.

Transcriptome expression data as FPKM values genome-wide is available in the Gene Expression Omnibus (GEO) at accession GSE133178. 
The genome annotation file and transcript and protein predictions are available from WormBase release 14 (WBPS14) onwards as accession Halicephalobus_mephisto_prjna528747.

\section{References}

1 Borgonie, G. et al. Nematoda from the terrestrial deep subsurface of South Africa. Nature 474, 79-82, doi:10.1038/nature09974 (2011).

2 Lau, M. C. Y. et al. An oligotrophic deep-subsurface community dependent on syntrophy is dominated by sulfur-driven autotrophic denitrifiers. P Natl Acad Sci USA 113, E7927E7936, doi:10.1073/pnas.1612244113 (2016).

3 Magnabosco, C. et al. Fluctuations in populations of subsurface methane oxidizers in coordination with changes in electron acceptor availability. FEMS Microbiol Ecol 94, doi:10.1093/femsec/fiy089 (2018).

4 Lau, M. C. Y. et al. Phylogeny and phylogeography of functional genes shared among seven terrestrial subsurface metagenomes reveal N-cycling and microbial evolutionary relationships. Front Microbiol 5, doi:ARTN 531

10.3389/fmicb.2014.00531 (2014).

5 Borgonie, G. et al. Eukaryotic opportunists dominate the deep-subsurface biosphere in South Africa. Nat Commun 6, 8952, doi:10.1038/ncomms9952 (2015).

6 Rödelsperger, C., Streit, A. \& Sommer, R. J. in eLS (John Wiley \& Sons, Ltd, 2013).

7 Wasmuth, J., Schmid, R., Hedley, A. \& Blaxter, M. On the extent and origins of genic novelty in the phylum Nematoda. PLoS Negl Trop Dis 2, e258, doi:10.1371/journal.pntd.0000258 (2008).

8 Coghlan, A. Nematode genome evolution. WormBook, 1-15, doi:10.1895/wormbook.1.15.1 (2005).

9 Borgonie, G. et al. Refuge from predation, the benefit of living in an extreme acidic environment? Biol Bull 219, 268-276, doi:10.1086/BBLv219n3p268 (2010).

10 Hunt, V. L. et al. The genomic basis of parasitism in the Strongyloides clade of nematodes. Nat Genet 48, 299-307, doi:10.1038/ng.3495 (2016).

11 Yin, D. et al. Rapid genome shrinkage in a self-fertile nematode reveals sperm competition proteins. Science 359, 55-+, doi:10.1126/science.aao0827 (2018).

12 Lespinet, O., Wolf, Y. I., Koonin, E. V. \& Aravind, L. The role of lineage-specific gene family expansion in the evolution of eukaryotes. Genome Res 12, 1048-1059, doi:10.1101/gr.174302 (2002).

13 Parra, G., Bradnam, K. \& Korf, I. CEGMA: a pipeline to accurately annotate core genes in eukaryotic genomes. Bioinformatics 23, 1061-1067, doi:10.1093/bioinformatics/btm071 (2007).

14 Parra, G., Bradnam, K., Ning, Z., Keane, T. \& Korf, I. Assessing the gene space in draft genomes. Nucleic Acids Res 37, 289-297, doi:10.1093/nar/gkn916 (2009).

15 Lowe, T. M. \& Eddy, S. R. tRNAscan-SE: A program for improved detection of transfer RNA genes in genomic sequence. Nucleic Acids Research 25, 955-964, doi:DOI 10.1093/nar/25.5.955 (1997). 
16 Simao, F. A., Waterhouse, R. M., Ioannidis, P., Kriventseva, E. V. \& Zdobnov, E. M. BUSCO: assessing genome assembly and annotation completeness with single-copy orthologs. Bioinformatics 31, 3210-3212, doi:10.1093/bioinformatics/btv351 (2015).

17 Blaxter, M. Nematodes: the worm and its relatives. PLoS Biol 9, e1001050, doi:10.1371/journal.pbio.1001050 (2011).

18 Srinivasan, J. et al. The Draft Genome and Transcriptome of Panagrellus redivivus Are Shaped by the Harsh Demands of a Free-Living Lifestyle. Genetics 193, 1279-+, doi:10.1534/genetics.112.148809 (2013).

19 Wheeler, T. J. \& Eddy, S. R. nhmmer: DNA homology search with profile HMMs. Bioinformatics 29, 2487-2489, doi:10.1093/bioinformatics/btt403 (2013).

20 Holt, C. \& Yandell, M. MAKER2: an annotation pipeline and genome-database management tool for second-generation genome projects. BMC Bioinformatics 12, 491, doi:10.1186/1471-2105-12-491 (2011).

21 Trapnell, C. et al. Differential gene and transcript expression analysis of RNA-seq experiments with TopHat and Cufflinks. Nat Protoc 7, 562-578, doi:10.1038/nprot.2012.016 (2012).

22 Pertea, M., Kim, D., Pertea, G. M., Leek, J. T. \& Salzberg, S. L. Transcript-level expression analysis of RNA-seq experiments with HISAT, StringTie and Ballgown. Nat Protoc 11, 1650-1667, doi:10.1038/nprot.2016.095 (2016).

23 Haas, B. J. et al. De novo transcript sequence reconstruction from RNA-seq using the Trinity platform for reference generation and analysis. Nat Protoc 8, 1494-1512, doi:10.1038/nprot.2013.084 (2013).

24 Chervitz, S. A. et al. Comparison of the complete protein sets of worm and yeast: orthology and divergence. Science 282, 2022-2028 (1998).

25 Thomas, J. H. Analysis of homologous gene clusters in Caenorhabditis elegans reveals striking regional cluster domains. Genetics 172, 127-143, doi:10.1534/genetics.104.040030 (2006).

26 Wang, Y., Coleman-Derr, D., Chen, G. \& Gu, Y. Q. OrthoVenn: a web server for genome wide comparison and annotation of orthologous clusters across multiple species. Nucleic Acids Res 43, W78-84, doi:10.1093/nar/gkv487 (2015).

27 Assimon, V. A., Gillies, A. T., Rauch, J. N. \& Gestwicki, J. E. Hsp70 Protein Complexes as Drug Targets. Curr Pharm Design 19, 404-417 (2013).

28 Sherman, M. Y. \& Gabai, V. L. Hsp70 in cancer: back to the future. Oncogene 34, 41534161, doi:10.1038/onc.2014.349 (2015).

29 Sorensen, J. G., Kristensen, T. N. \& Loeschcke, V. The evolutionary and ecological role of heat shock proteins. Ecol Lett 6, 1025-1037, doi:10.1046/j.1461-0248.2003.00528.x (2003).

30 Usman, M. G. et al. Molecular analysis of Hsp70 mechanisms in plants and their function in response to stress. Biotechnol Genet Eng 33, 26-39, doi:10.1080/02648725.2017.1340546 (2017).

31 Brunquell, J., Morris, S., Lu, Y., Cheng, F. \& Westerheide, S. D. The genome-wide role of HSF-1 in the regulation of gene expression in Caenorhabditis elegans. Bmc Genomics 17, doi:ARTN 559

10.1186/s12864-016-2837-5 (2016). 
32 Lakhotia, S. C., Srivastava, P. \& Prasanth, K. V. Regulation of heat shock proteins, Hsp70 and Hsp64, in heat-shocked Malpighian tubules of Drosophila melanogaster larvae. Cell Stress Chaperones 7, 347-356 (2002).

33 Prahlad, V., Cornelius, T. \& Morimoto, R. I. Regulation of the cellular heat shock response in Caenorhabditis elegans by thermosensory neurons. Science 320, 811-814, doi:10.1126/science.1156093 (2008).

34 Rodriguez, M., Snoek, L. B., De Bono, M. \& Kammenga, J. E. Worms under stress: C. elegans stress response and its relevance to complex human disease and aging. Trends Genet 29, 367-374, doi:10.1016/j.tig.2013.01.010 (2013).

35 Yu, A. et al. Roles of Hsp70s in Stress Responses of Microorganisms, Plants, and Animals. Biomed Res Int 2015, 510319, doi:10.1155/2015/510319 (2015).

36 Brocchieri, L., de Macario, E. C. \& Macario, A. J. L. hsp70 genes in the human genome: Conservation and differentiation patterns predict a wide array of overlapping and specialized functions. Bmc Evol Biol 8, doi:Artn 19

10.1186/1471-2148-8-19 (2008).

37 Gibbs, D. S., Anderson, G. L., Beuchat, L. R., Carta, L. K. \& Williams, P. L. Potential role of Diploscapter sp strain LKC25, a bacterivorous nematode from soil, as a vector of food-borne pathogenic bacteria to preharvest fruits and vegetables. Appl Environ Microb 71, 2433-2437, doi:10.1128/Aem.7.1.5.2433-2437.2005 (2005).

38 Chandler, A. C. Diploscapter coronata as a facultative parasite of man, with a general review of vertebrate parasitism by rhabditoid worms. Parasitology 30, 44-55, doi:10.1017/S0031182000010817 (2009).

39 Lemzina, L. V. \& Gagarin, V. G. I. o. B., Kyrghyzian Academy of Sciences, Chou pr. 265, Bishkek, Kyrghyzstan). New species of free-living nematodes from thermal waters in Kyrghyzstan. v. 3 (1994).

40 Yang, Z. Inference of selection from multiple species alignments. Curr Opin Genet Dev 12, 688-694 (2002).

41 Yang, Z. PAML 4: phylogenetic analysis by maximum likelihood. Mol Biol Evol 24, 1586-1591, doi:10.1093/molbev/msm088 (2007).

42 Yang, Z., Wong, W. S. \& Nielsen, R. Bayes empirical bayes inference of amino acid sites under positive selection. Mol Biol Evol 22, 1107-1118, doi:10.1093/molbev/msi097 (2005).

43 Perneger, T. V. What's wrong with Bonferroni adjustments. BMJ 316, 1236-1238 (1998).

44 Reuber, T. L. \& Ausubel, F. M. Isolation of arabidopsis genes that differentiate between resistance responses mediated by the RPS2 and RPM1 disease resistance genes. Plant Cell 8, 241-249, doi:DOI 10.1105/tpc.8.2.241 (1996).

45 Nitta, T. et al. IAN family critically regulates survival and development of T lymphocytes. PLoS Biol 4, e103, doi:10.1371/journal.pbio.0040103 (2006).

46 Wang, Z. \& Li, X. IAN/GIMAPs are conserved and novel regulators in vertebrates and angiosperm plants. Plant Signal Behav 4, 165-167 (2009).

47 Blaxter, M. \& Koutsovoulos, G. The evolution of parasitism in Nematoda. Parasitology 142 Suppl 1, S26-39, doi:10.1017/S0031182014000791 (2015).

48 Liu, C., Wang, T., Zhang, W. \& Li, X. Computational identification and analysis of immune-associated nucleotide gene family in Arabidopsis thaliana. J Plant Physiol 165, 777-787, doi:10.1016/j.jplph.2007.06.002 (2008). 
49 Lin, K. et al. Single Nucleus Genome Sequencing Reveals High Similarity among Nuclei of an Endomycorrhizal Fungus. Plos Genetics 10, doi:ARTN e1004078

10.1371/journal.pgen.1004078 (2014).

50 Nitta, T. \& Takahama, Y. The lymphocyte guard-IANs: regulation of lymphocyte survival by IAN/GIMAP family proteins. Trends Immunol 28, 58-65, doi:10.1016/j.it.2006.12.002 (2007).

51 Steiner, G. Opuscula miscellanea nematologica. IX. Proceedings of the Helminthological Society of Washington 9, 32-34 (1942).

52 McGill, L. M., Fitzpatrick, D. A., Pisani, D. \& Burnell, A. M. Estimation of phylogenetic divergence times in Panagrolaimidae and other nematodes using relaxed molecular clocks calibrated with insect and crustacean fossils. Nematology 19, 899-+, doi:10.1163/15685411-00003096 (2017).

53 Okimoto, R., Macfarlane, J. L., Clary, D. O. \& Wolstenholme, D. R. The mitochondrial genomes of two nematodes, Caenorhabditis elegans and Ascaris suum. Genetics 130, 471-498 (1992).

54 Parkinson, J. \& Blaxter, M. SimiTri--visualizing similarity relationships for groups of sequences. Bioinformatics 19, 390-395 (2003).

55 Friedman, R. \& Ely, B. Codon usage methods for horizontal gene transfer detection generate an abundance of false positive and false negative results. Curr Microbiol 65, 639-642, doi:10.1007/s00284-012-0205-5 (2012).

56 Gallegos, M. E. \& Bargmann, C. I. Mechanosensory neurite termination and tiling depend on SAX-2 and the SAX-1 kinase. Neuron 44, 239-249, doi:10.1016/j.neuron.2004.09.021 (2004).

57 Wormbase. (www.wormbase.org).

58 Mizobuchi, N. et al. ARMET is a soluble ER protein induced by the unfolded protein response via ERSE-II element. Cell Struct Funct 32, 41-50, doi:DOI 10.1247/csf.07001 (2007).

59 Glembotski, C. C. et al. Mesencephalic astrocyte-derived neurotrophic factor protects the heart from ischemic damage and is selectively secreted upon sarco/endoplasmic reticulum calcium depletion. J Biol Chem 287, 25893-25904, doi:10.1074/jbc.M112.356345 (2012).

60 Apostolou, A., Shen, Y., Liang, Y., Luo, J. \& Fang, S. Armet, a UPR-upregulated protein, inhibits cell proliferation and ER stress-induced cell death. Exp Cell Res 314, 2454-2467, doi:10.1016/j.yexcr.2008.05.001 (2008).

61 Zhang, Z. et al. MANF protects dopamine neurons and locomotion defects from a human alpha-synuclein induced Parkinson's disease model in C. elegans by regulating ER stress and autophagy pathways. Exp Neurol 308, 59-71, doi:10.1016/j.expneurol.2018.06.016 (2018).

62 Cai, J. et al. Bax inhibitor-1 from orange spotted grouper, Epinephelus coioides involved in viral infection. Fish Shellfish Immunol 78, 91-99, doi:10.1016/j.fsi.2018.04.020 (2018).

63 Sano, R. \& Reed, J. C. ER stress-induced cell death mechanisms. Biochim Biophys Acta 1833, 3460-3470, doi:10.1016/j.bbamcr.2013.06.028 (2013).

64 Cutter, A. D. Divergence times in Caenorhabditis and Drosophila inferred from direct estimates of the neutral mutation rate. Mol Biol Evol 25, 778-786, doi:10.1093/molbev/msn024 (2008). 
65 Stein, L. D. et al. The genome sequence of Caenorhabditis briggsae: a platform for comparative genomics. PLoS Biol 1, E45, doi:10.1371/journal.pbio.0000045 (2003).

66 Borgonie, G. et al. New ecosystems in the deep subsurface follow the flow of water driven by geological activity. Sci Rep 9, 3310, doi:10.1038/s41598-019-39699-w (2019).

67 Opperman, C. H. et al. Sequence and genetic map of Meloidogyne hapla: A compact nematode genome for plant parasitism. Proc Natl Acad Sci U S A 105, 14802-14807, doi:10.1073/pnas.0805946105 (2008).

68 Abad, P. et al. Genome sequence of the metazoan plant-parasitic nematode Meloidogyne incognita. Nat Biotechnol 26, 909-915, doi:10.1038/nbt. 1482 (2008).

69 Fradin, H. et al. Genome Architecture and Evolution of a Unichromosomal Asexual Nematode. Curr Biol 27, 2928-2939 e2926, doi:10.1016/j.cub.2017.08.038 (2017).

70 Kajitani, R. et al. Efficient de novo assembly of highly heterozygous genomes from whole-genome shotgun short reads. Genome Research 24, 1384-1395, doi:10.1101/gr.170720.113 (2014).

71 Treangen, T. J. \& Salzberg, S. L. Repetitive DNA and next-generation sequencing: computational challenges and solutions. Nat Rev Genet 13, 36-46, doi:10.1038/nrg3117 (2011).

72 Wicky, C. et al. Telomeric repeats (TTAGGC)n are sufficient for chromosome capping function in Caenorhabditis elegans. Proc Natl Acad Sci U S A 93, 8983-8988 (1996).

73 Zhang, J., Kobert, K., Flouri, T. \& Stamatakis, A. PEAR: a fast and accurate Illumina Paired-End reAd mergeR. Bioinformatics 30, 614-620, doi:10.1093/bioinformatics/btt593 (2014).

74 Hollien, J. \& Weissman, J. S. Decay of endoplasmic reticulum-localized mRNAs during the unfolded protein response. Science 313, 104-107, doi:10.1126/science.1129631 (2006).

75 Maurel, M., Chevet, E., Tavernier, J. \& Gerlo, S. Getting RIDD of RNA: IRE1 in cell fate regulation. Trends Biochem Sci 39, 245-254, doi:10.1016/j.tibs.2014.02.008 (2014).

76 Krebs, R. A. \& Feder, M. E. Deleterious consequences of Hsp70 overexpression in Drosophila melanogaster larvae. Cell Stress Chaperones 2, 60-71 (1997).

77 Krebs, R. A. \& Loeschcke, V. Effects of Exposure to Short-Term Heat-Stress on Fitness Components in Drosophila-Melanogaster. J Evolution Biol 7, 39-49, doi:DOI 10.1046/j.1420-9101.1994.7010039.x (1994).

78 Williams, K. D., Helin, A. B., Posluszny, J., Roberts, S. P. \& Feder, M. E. Effect of heat shock, pretreatment and hsp70 copy number on wing development in Drosophila melanogaster. Mol Ecol 12, 1165-1177 (2003).

79 Sorensen, J. G., Dahlgaard, J. \& Loeschcke, V. Genetic variation in thermal tolerance among natural populations of Drosophila buzzatii: down regulation of Hsp70 expression and variation in heat stress resistance traits. Funct Ecol 15, 289-296, doi:DOI 10.1046/j.1365-2435.2001.00525.x (2001).

80 Narum, S. R., Campbell, N. R., Meyer, K. A., Miller, M. R. \& Hardy, R. W. Thermal adaptation and acclimation of ectotherms from differing aquatic climates. Mol Ecol 22, 3090-3097, doi:10.1111/mec.12240 (2013).

81 Magnabosco, C. et al. Comparisons of the composition and biogeographic distribution of the bacterial communities occupying South African thermal springs with those inhabiting deep subsurface fracture water. Front Microbiol 5, doi:ARTN 679

10.3389/fmicb.2014.00679 (2014). 
82 Simkus, D. N. et al. Variations in microbial carbon sources and cycling in the deep continental subsurface. Geochimica et Cosmochimica Acta 173, 264-283, doi:https://doi.org/10.1016/j.gca.2015.10.003 (2016).

83 Powell, D. et al. The genome of the oyster Saccostrea offers insight into the environmental resilience of bivalves. DNA Res 25, 655-665, doi:10.1093/dnares/dsy032 (2018).

84 Zhang, G. et al. The oyster genome reveals stress adaptation and complexity of shell formation. Nature 490, 49-54, doi:10.1038/nature11413 (2012).

85 Lim, C. K. et al. First human case of fatal Halicephalobus gingivalis meningoencephalitis in Australia. J Clin Microbiol 53, 1768-1774, doi:10.1128/JCM.00032-15 (2015).

86 Brenner, S. The genetics of Caenorhabditis elegans. Genetics 77, 71-94 (1974).

87 Corvelo, A., Clarke, W. E., Robine, N. \& Zody, M. C. taxMaps: comprehensive and highly accurate taxonomic classification of short-read data in reasonable time. Genome Res 28, 751-758, doi:10.1101/gr.225276.117 (2018).

$88 \mathrm{Li}, \mathrm{R}$. et al. De novo assembly of human genomes with massively parallel short read sequencing. Genome Res 20, 265-272, doi:10.1101/gr.097261.109 (2010).

89 Smit, A. F. A., Hubley, R. \& Green, P. RepeatMasker Open-4.0. http://www.repeatmasker.org.

90 Eddy, S. R. Accelerated Profile HMM Searches. PLoS Comput Biol 7, e1002195, doi:10.1371/journal.pcbi.1002195 (2011).

91 Wheeler, T. J. et al. Dfam: a database of repetitive DNA based on profile hidden Markov models. Nucleic Acids Res 41, D70-82, doi:10.1093/nar/gks1265 (2013).

92 Frazee, A. C. et al. Ballgown bridges the gap between transcriptome assembly and expression analysis. Nat Biotechnol 33, 243-246, doi:10.1038/nbt.3172 (2015).

93 Eddy, S. R. Profile hidden Markov models. Bioinformatics 14, 755-763 (1998).

94 Fischer, S. et al. Using OrthoMCL to assign proteins to OrthoMCL-DB groups or to cluster proteomes into new ortholog groups. Curr Protoc Bioinformatics Chapter 6, Unit 6 12 11-19, doi:10.1002/0471250953.bi0612s35 (2011).

95 Li, L., Stoeckert, C. J., Jr. \& Roos, D. S. OrthoMCL: identification of ortholog groups for eukaryotic genomes. Genome Res 13, 2178-2189, doi:10.1101/gr.1224503 (2003).

96 Camacho, C. et al. BLAST+: architecture and applications. BMC Bioinformatics 10, 421, doi:10.1186/1471-2105-10-421 (2009).

97 Ekseth, O. K., Kuiper, M. \& Mironov, V. orthAgogue: an agile tool for the rapid prediction of orthology relations. Bioinformatics 30, 734-736, doi:10.1093/bioinformatics/btt582 (2014).

98 Edgar, R. C. MUSCLE: multiple sequence alignment with high accuracy and high throughput. Nucleic Acids Res 32, 1792-1797, doi:10.1093/nar/gkh340 (2004).

99 Capella-Gutierrez, S., Silla-Martinez, J. M. \& Gabaldon, T. trimAl: a tool for automated alignment trimming in large-scale phylogenetic analyses. Bioinformatics 25, 1972-1973, doi:10.1093/bioinformatics/btp348 (2009).

100 Darriba, D., Taboada, G. L., Doallo, R. \& Posada, D. ProtTest 3: fast selection of best-fit models of protein evolution. Bioinformatics 27, 1164-1165, doi:10.1093/bioinformatics/btr088 (2011).

101 Klopfenstein, D. V. et al. GOATOOLS: A Python library for Gene Ontology analyses. Sci Rep 8, 10872, doi:10.1038/s41598-018-28948-z (2018). 
102 Jones, P. et al. InterProScan 5: genome-scale protein function classification. Bioinformatics 30, 1236-1240, doi:10.1093/bioinformatics/btu031 (2014).

103 Katoh, K., Misawa, K., Kuma, K. \& Miyata, T. MAFFT: a novel method for rapid multiple sequence alignment based on fast Fourier transform. Nucleic Acids Res 30, 3059-3066 (2002).

104 Huelsenbeck, J. P. \& Ronquist, F. MRBAYES: Bayesian inference of phylogenetic trees. Bioinformatics 17, 754-755 (2001).

105 Stamatakis, A. RAxML version 8: a tool for phylogenetic analysis and post-analysis of large phylogenies. Bioinformatics 30, 1312-1313, doi:10.1093/bioinformatics/btu033 (2014).

106 Yang, Z. PAML: a program package for phylogenetic analysis by maximum likelihood. Comput Appl Biosci 13, 555-556 (1997).

107 Yang, Z. Likelihood ratio tests for detecting positive selection and application to primate lysozyme evolution. Mol Biol Evol 15, 568-573, doi:10.1093/oxfordjournals.molbev.a025957 (1998).

108 Chevreux, B. et al. Using the miraEST assembler for reliable and automated mRNA transcript assembly and SNP detection in sequenced ESTs. Genome Research 14, 1147 1159, doi:10.1101/gr.1917404 (2004). 


\begin{tabular}{|c|c|c|}
\hline & RepeatMasker & nhmmer \\
\hline Retrotransposons & 3.8 & 8.8 \\
\hline DNA Transposons & 8.8 & 23.6 \\
\hline Helitrons & 0.0 & 44.3 \\
\hline Unclassified & 87.3 & 23.3 \\
\hline
\end{tabular}

\section{Table 1. Repetitive element composition of the $\boldsymbol{H}$.}

mephisto genome. The percentage of repeats belonging to each class are shown.

\begin{tabular}{|c|c|c|c|c|}
\hline & H. mephisto & C. elegans & M. hapla & P. redivivus \\
\hline $\begin{array}{l}\text { Assembly size } \\
(\mathrm{Mb})\end{array}$ & 61.4 & 100 & 53.0 & 64.4 \\
\hline N50 (kb) & 313 & 17,494 & 38 & 262 \\
\hline \# of scaffolds $(\mathrm{kb})$ & 880 & 7 & 3,452 & 940 \\
\hline longest (kb) & 2,546 & 20,924 & 360 & 2,280 \\
\hline shortest (kb) & 1 & 13.8 & 0.7 & 0.4 \\
\hline $\begin{array}{l}\text { Protein-coding } \\
\text { loci } \\
\text { (nonredundant) }\end{array}$ & 16,186 & 19,922 & 14,420 & 24,249 \\
\hline $\begin{array}{l}\text { Potential non- } \\
\text { coding loci }\end{array}$ & 1,023 & 703 & - & - \\
\hline $\begin{array}{l}\text { Average Intron } \\
\text { length (bp) }\end{array}$ & 473 & 320 & 153 & 163 \\
\hline $\begin{array}{l}\text { Average Exon } \\
\text { length (bp) }\end{array}$ & 332 & 202 & 171 & 288 \\
\hline Transcripts & 34,605 & 33,303 & 16,676 & 26,372 \\
\hline $\begin{array}{l}\text { Repetitive content } \\
\qquad(\%)\end{array}$ & 24.3 & 12.7 & 18.3 & 7.1 \\
\hline GC content $(\%)$ & 32.1 & 35.4 & 27.4 & 44.3 \\
\hline
\end{tabular}

Table 2. Genome and transcriptome data for $H$. mephisto and comparison to $C$. elegans, $M$.

hapla, and P. redivivus. Data for C. elegans and P. redivivus from Srinivasan et al. 2013 and for $M$. hapla from Foth et al., 2014. 


\begin{tabular}{|c|l|c|c|c|c|c|c|}
\hline \multicolumn{2}{|c|}{ Branch } & $\begin{array}{c}\omega_{2} \\
\text { positive } \\
\text { selection) }\end{array}$ & $\begin{array}{c}\omega_{1} \\
\text { (purifying } \\
\text { selection) }\end{array}$ & $\begin{array}{c}\text { Fraction Sites } \\
\text { under } \omega_{2}\end{array}$ & $\begin{array}{c}\text { Fraction Sites } \\
\text { under } \omega_{1}\end{array}$ & $\begin{array}{c}\text { LRT } \\
\text { Statistic } \\
(2 \Delta \operatorname{lnL})\end{array}$ & p-value \\
\hline A & HMEPH-07507-RA $\Delta$ & 3.58 & 0.17 & 0.12 & 0.81 & 1.98 & 0.15939 \\
\hline B & HMEPH-08905-RA $\Delta$ & 2.94 & 0.17 & 0.05 & 0.88 & 1.6 & 0.205903 \\
\hline C & HMEPH-16538-RA & 1.54 & 0.17 & 0.11 & 0.82 & 0.22 & 0.63904 \\
\hline D & HMEPH-09138-RA $\Delta$ & 94.01 & 0.17 & 0.01 & 0.92 & 2.4 & 0.121335 \\
\hline E & HMEPH-09222-RA & 86.51 & 0.17 & 0.24 & 0.68 & 104.48 & $\mathbf{1 . 5 9 e - 2 4}$ \\
\hline F & Long Branch & 196.81 & 0.17 & 0.89 & 0.04 & 4.16 & 0.041389 \\
\hline G & HMEPH-08969-RA $\Delta$ & 999 & 0.17 & 0.08 & 0.84 & 8.12 & 0.004378 \\
\hline H & HMEPH-13099-RA & 18.27 & 0.17 & 0.09 & 0.84 & 11.68 & $\mathbf{0 . 0 0 0 6 3 2}$ \\
\hline I & HMEPH-12059-RA $\Delta \Delta$ & 1 & 0.17 & 0.75 & 0.18 & 0 & 1 \\
\hline J & dp_WR25_05901J.1 & 12.04 & 0.17 & 0.31 & 0.62 & 14.46 & $\mathbf{0 . 0 0 0 1 4 3}$ \\
\hline K & $\begin{array}{l}\text { Diploscapter Cluster III } \\
\text { root }\end{array}$ & 999 & 0.17 & 0.13 & 0.80 & 26.36 & $\mathbf{2 . 8 3 e - 7}$ \\
\hline L & DCO_000646 & 1 & 0.17 & 0 & 0.93 & 0 & 1 \\
\hline M & dp_WR25_09899A.1 & 1 & 0.17 & 0.40 & 0.52 & 0 & 1 \\
\hline
\end{tabular}

Table 3. Branch-site analysis of $\mathrm{dN} / \mathrm{dS}$ ratios $(\omega)$ from tree in Figure $2 B$. Bold $p$-values are statistically significant after correcting for multiple hypothesis testing $(\mathrm{p}=0.0038)$. Likelihood ratio test (LRT) statistic applied to a $\chi^{2}$ table with $\mathrm{df}=1$, critical values $3.84(5 \%)$ and $6.63(1 \%)$. Note that $\omega_{2}$ is constrained to be greater than or equal to 1 (positive to neutral selection). $\Delta$, expression increased $1.5 \mathrm{x}$ under heat stress. $\Delta \Delta$, expression increased $2 \mathrm{x}$ under heat stress.

\begin{tabular}{|l|l|l|l|l|l|}
\hline GO identifier & name & $\begin{array}{l}\text { ratio in } \\
\text { study }\end{array}$ & $\begin{array}{l}\text { ratio in } \\
\text { population }\end{array}$ & p_uncorrected & p_bonferroni \\
\hline GO:0008234 & $\begin{array}{l}\text { cysteine-type peptidase } \\
\text { activity }\end{array}$ & $19 / 649$ & $133 / 28062$ & $4.83 \mathrm{E}-07$ & 0.0014 \\
\hline GO:0042302 & $\begin{array}{l}\text { structural constituent of } \\
\text { cuticle }\end{array}$ & $13 / 649$ & $89 / 28062$ & $9.91 \mathrm{E}-07$ & 0.0029 \\
\hline GO:0004587 & $\begin{array}{l}\text { ornithine-oxo-acid } \\
\text { transaminase activity }\end{array}$ & $3 / 649$ & $3 / 28062$ & $1.23 \mathrm{E}-05$ & 0.0362 \\
\hline
\end{tabular}

Table 4. GO terms enriched in $\boldsymbol{H}$. mephisto genes downregulated under heat stress. 


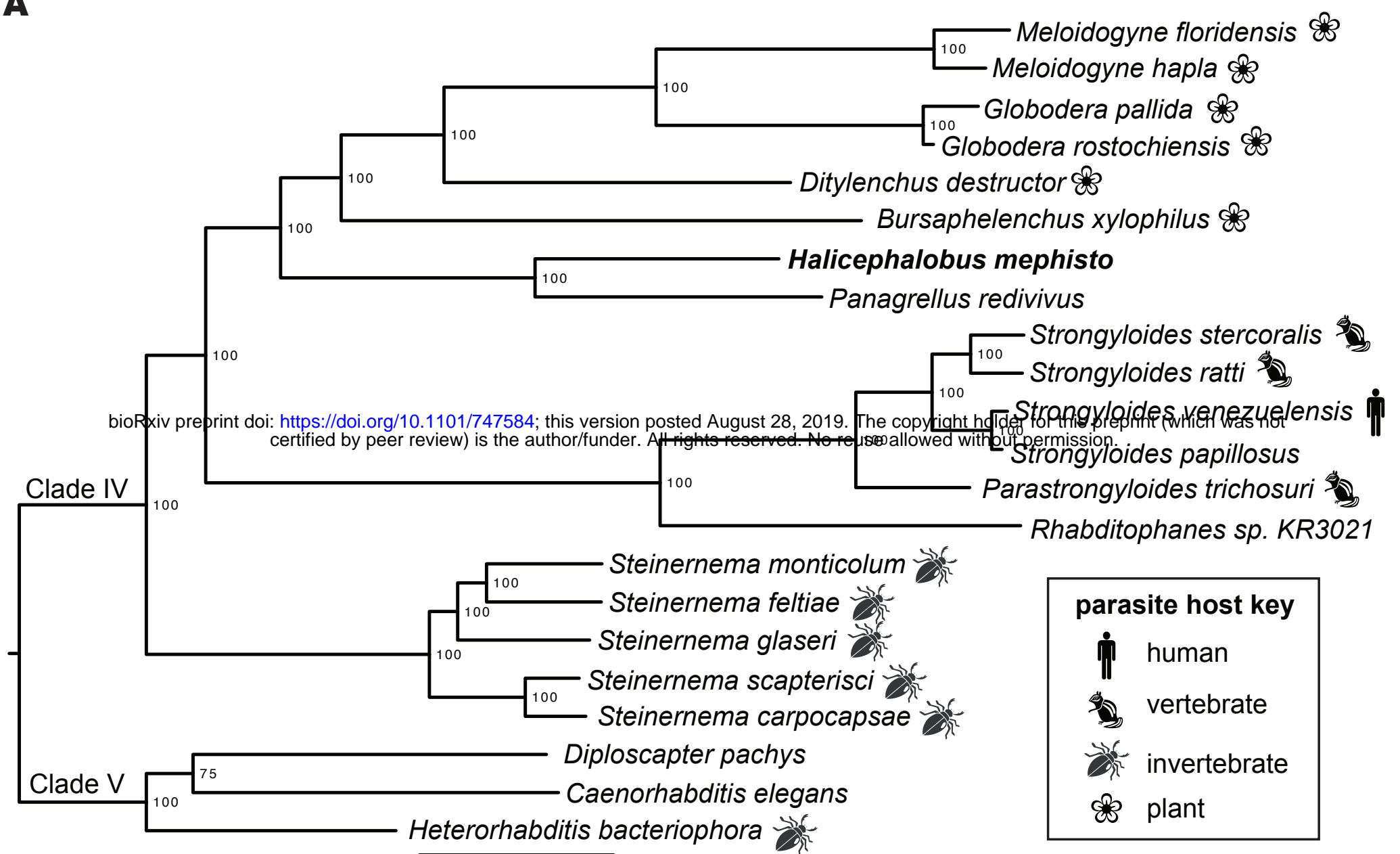

B

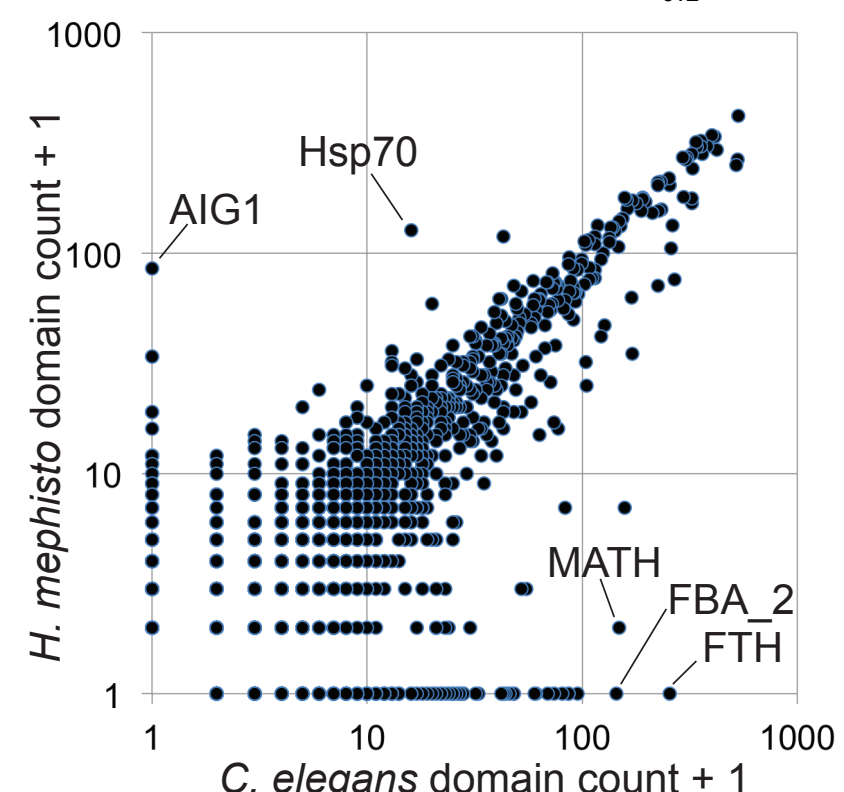

D

Caenorhabditis elegans

\section{Drosophila melanogaster}

C

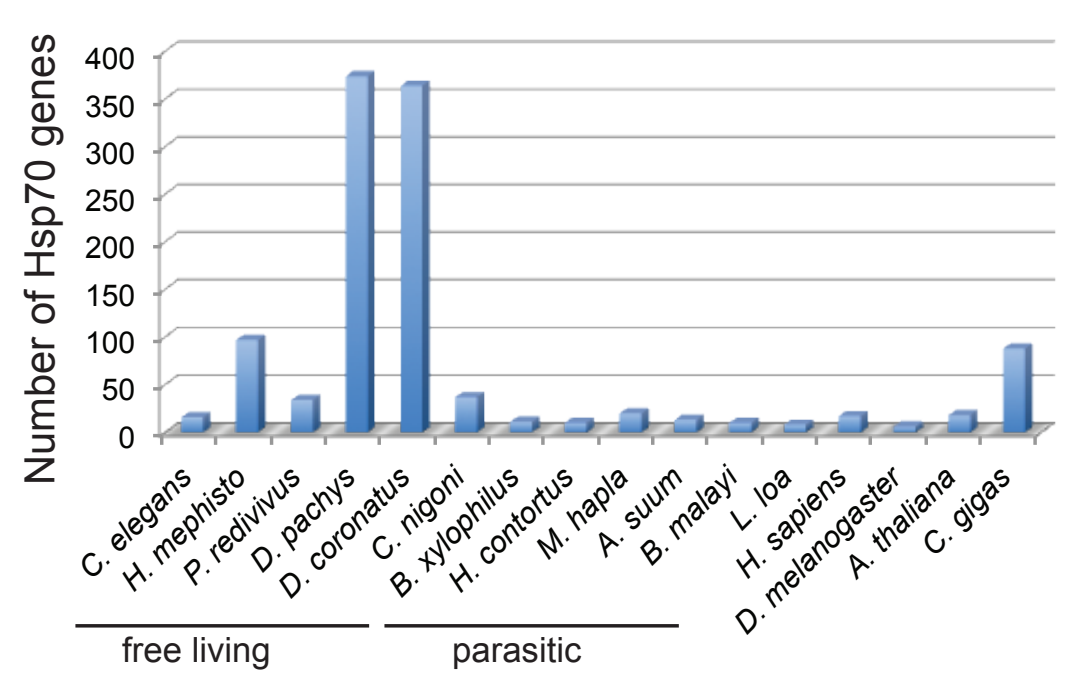

Halicephalobus mephisto

417

90

Panagrellus redivivus

350

1563

46

2164

761

3233 


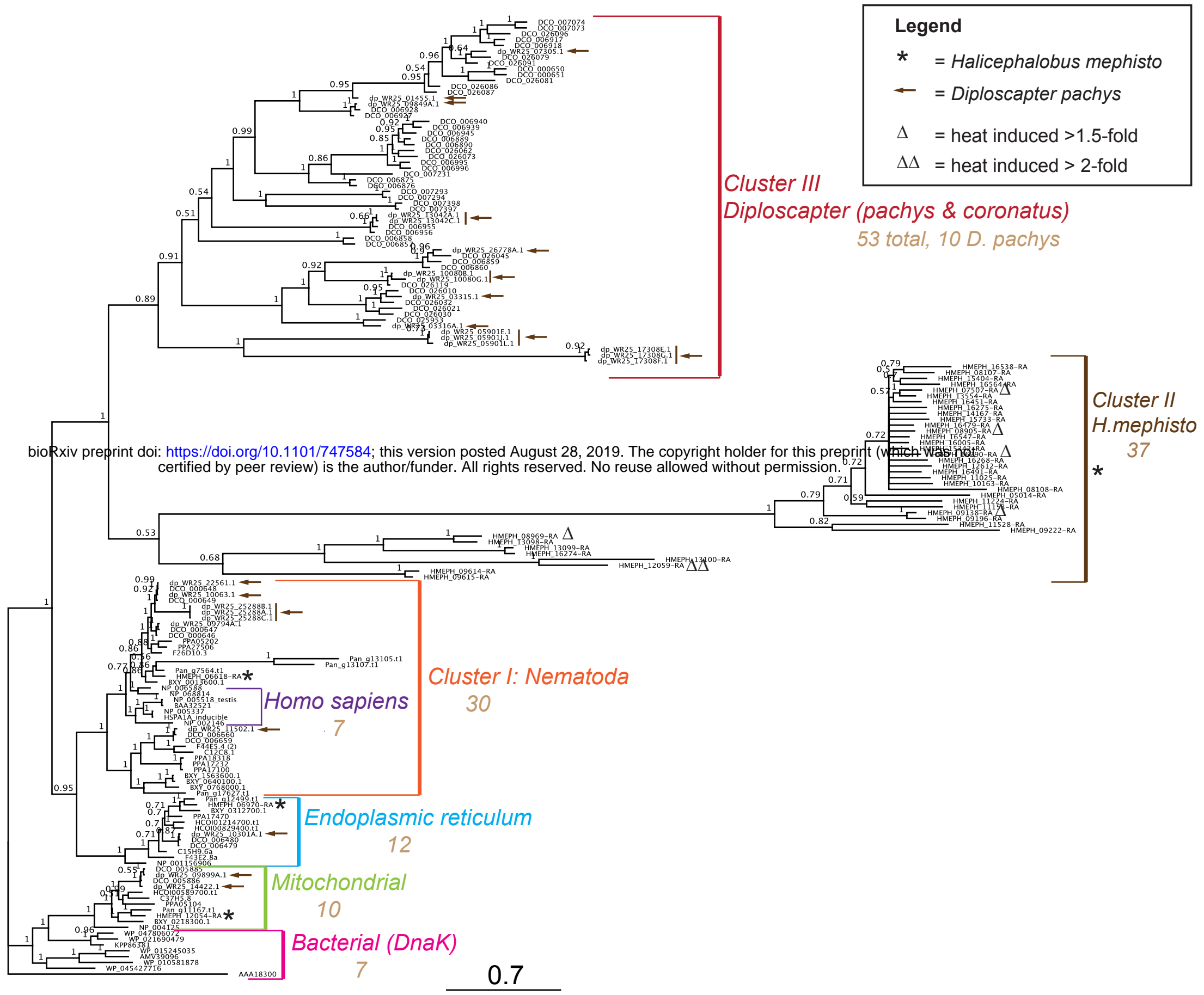

B

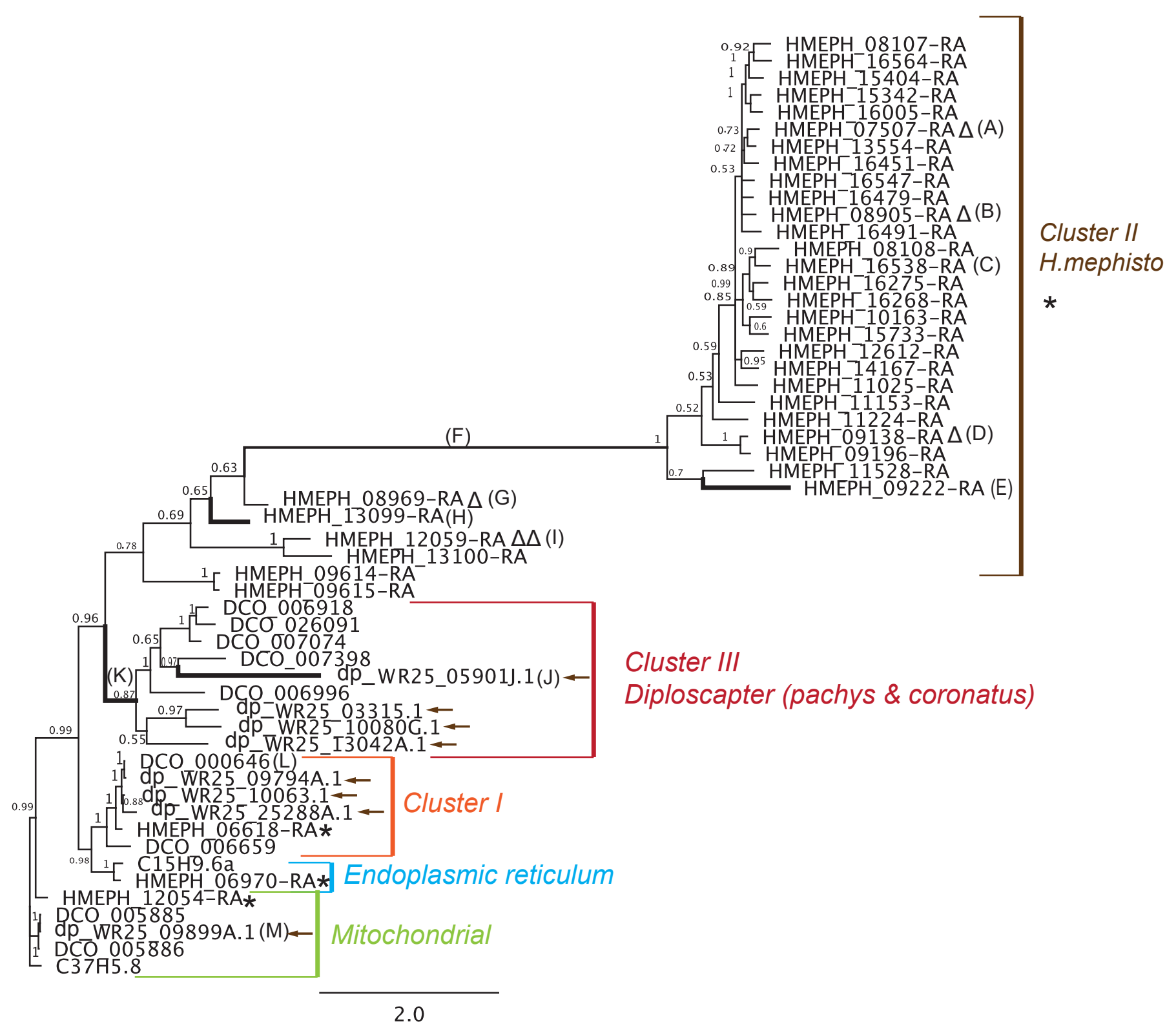




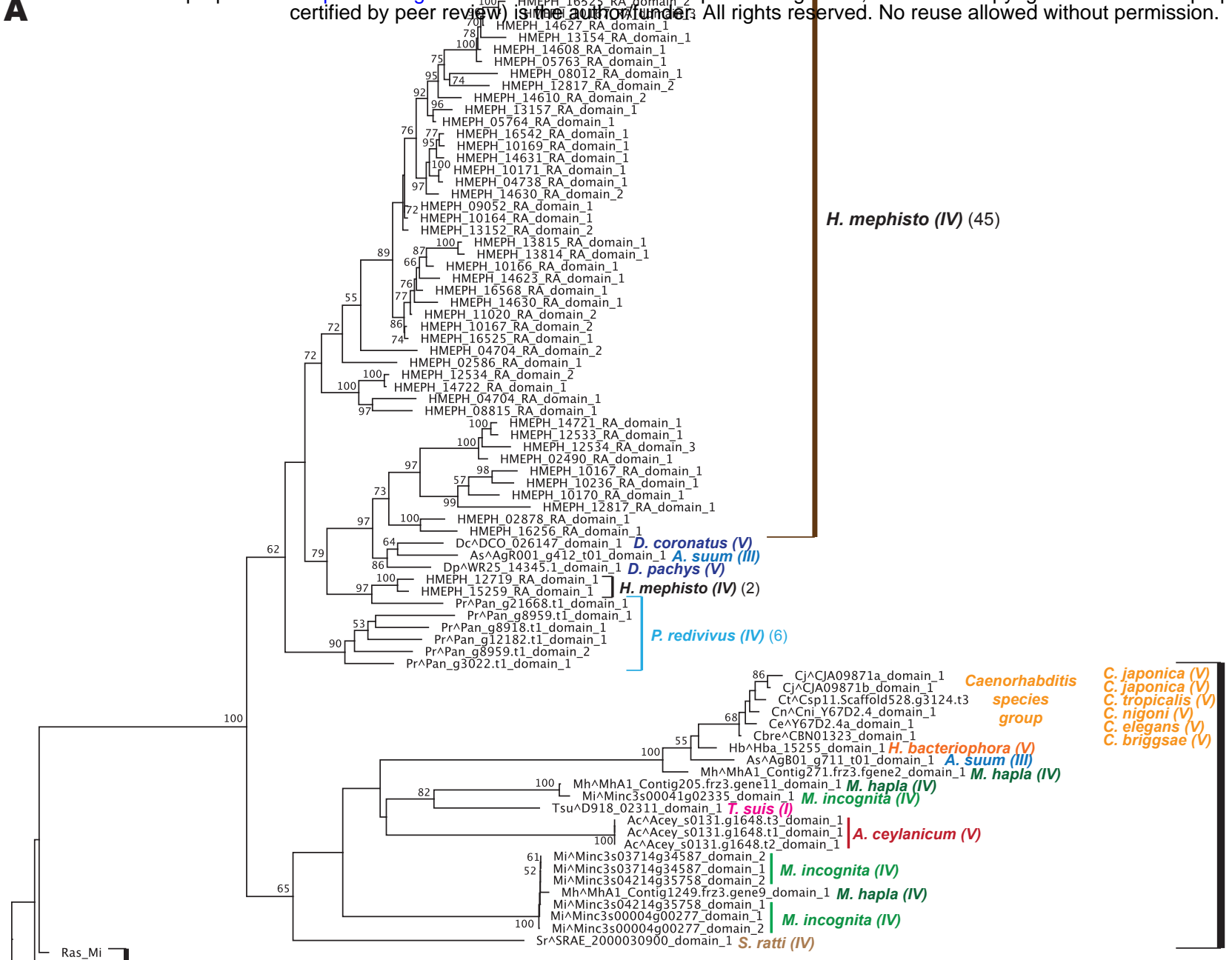

AIG1-like (56)

MTG1-related (23)

B

1.0

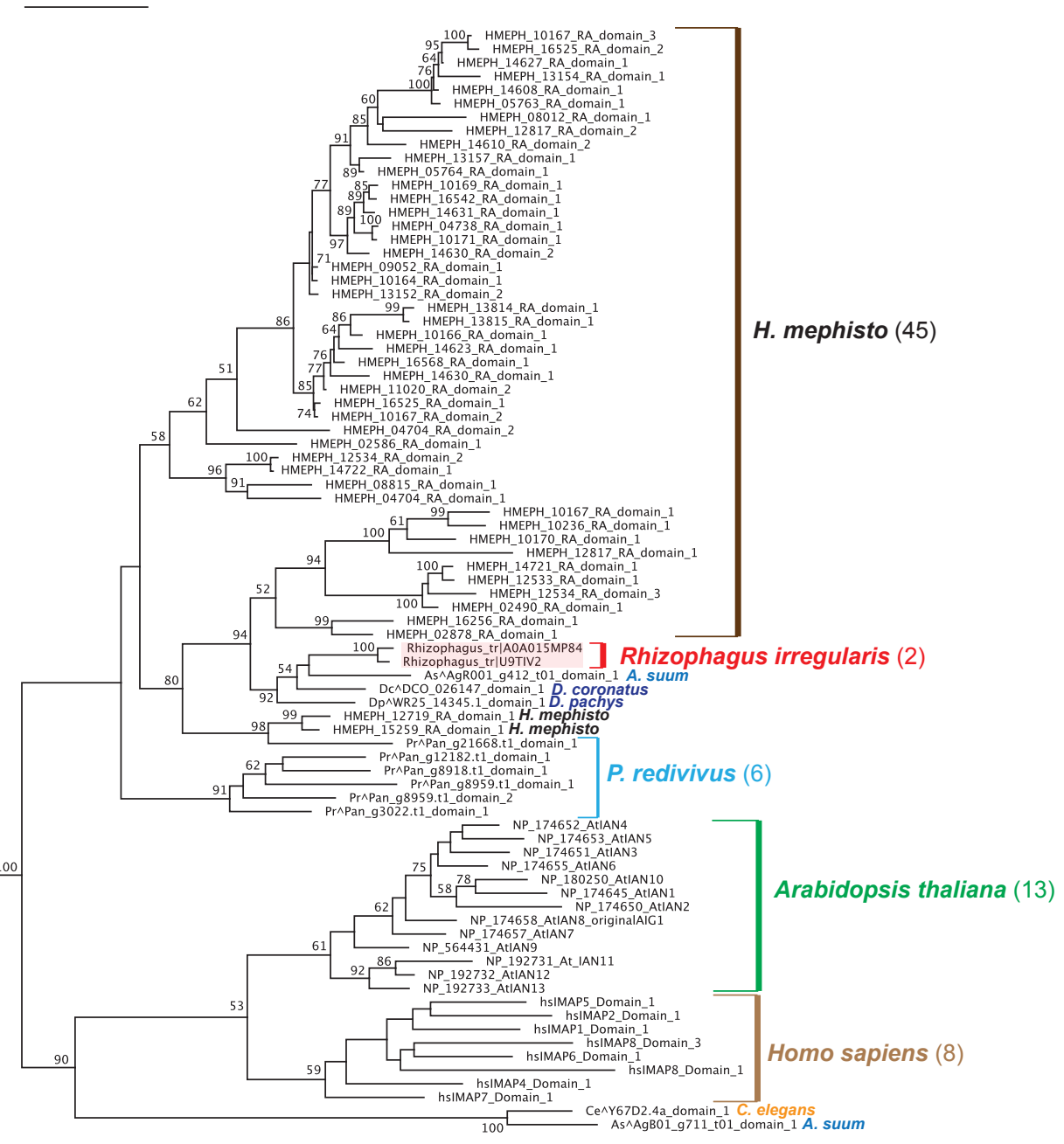

IAN / GIMAP

(21) 
A

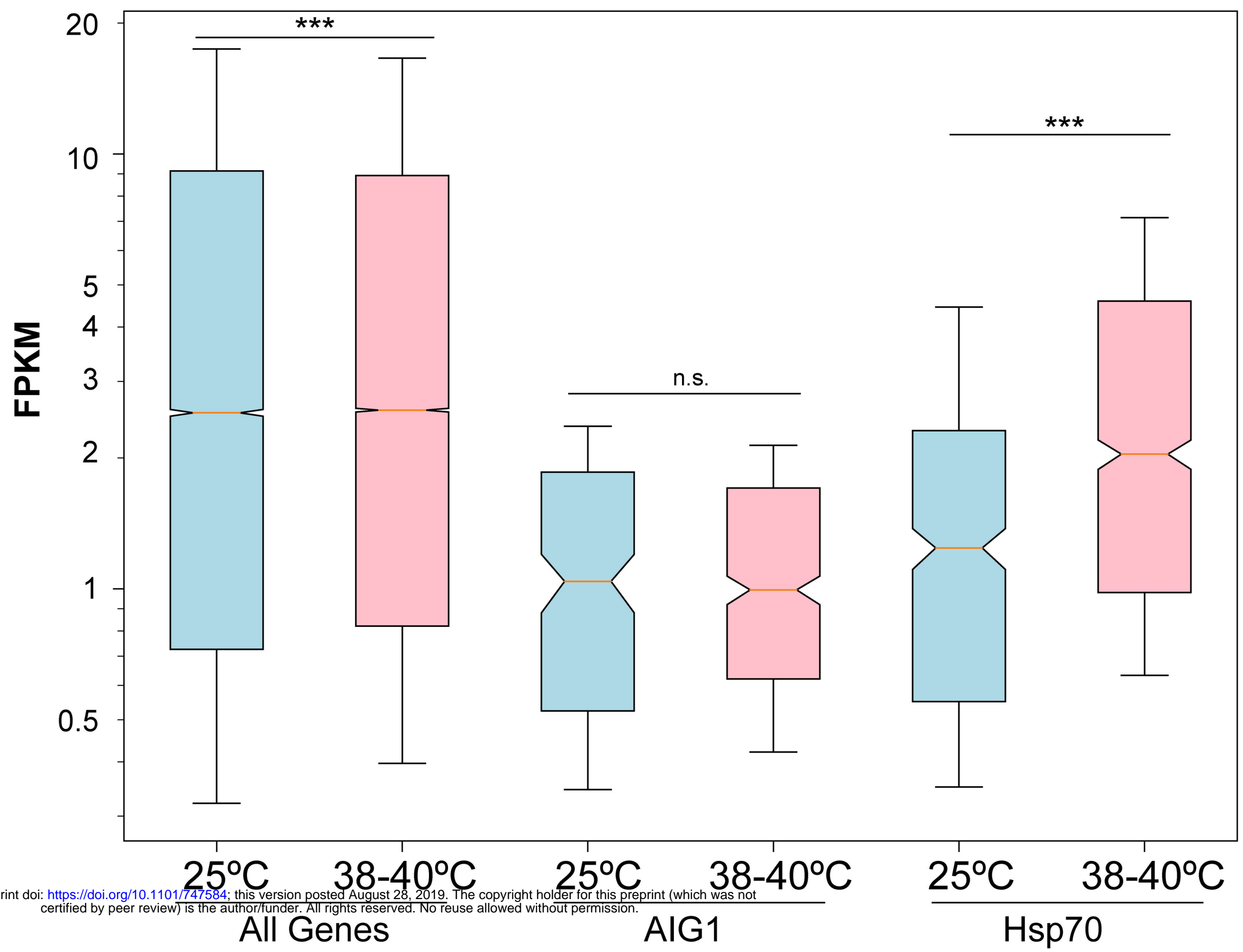

B

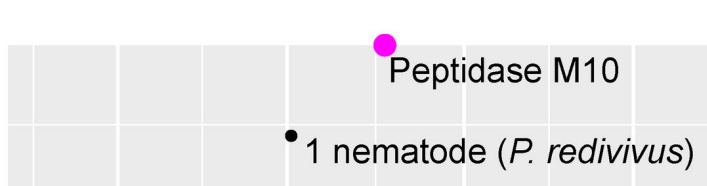

Statistically downregulated: 675 transcripts

Statistically upregulated: 285 transcripts

$1 e-6$

$\sum_{\frac{1}{\sigma}}^{\frac{0}{2}} 1 \mathrm{e}-4$

$1 \mathrm{e}-2$

1.

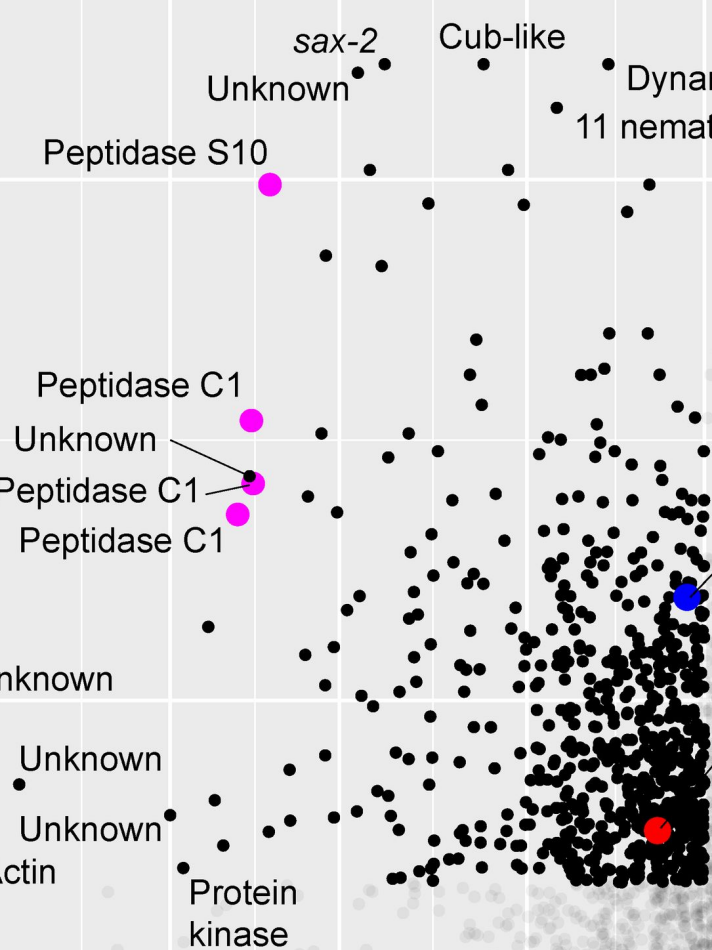

- sax-2
- 16 nematodes

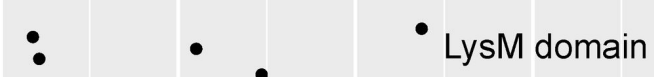<smiles>C[13CH][13CH3]</smiles>

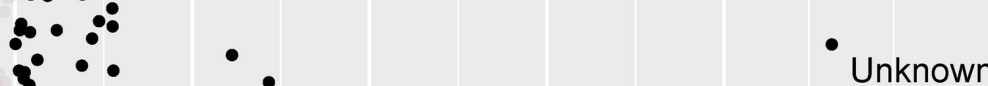

Unknown

ARMET

- Unknown 
bioRxiv preprint doi: https://doi.org/10.1101/747584; this version posted August 28, 2019. The copyright holder for this preprint (which was not certified by peer review) is the author/funder. All rights reserved. No reuse allowed without permission.

* = Halicephalobus mephisto

\section{Cluster III}

Diploscapter (pachys \& coronatus)

59

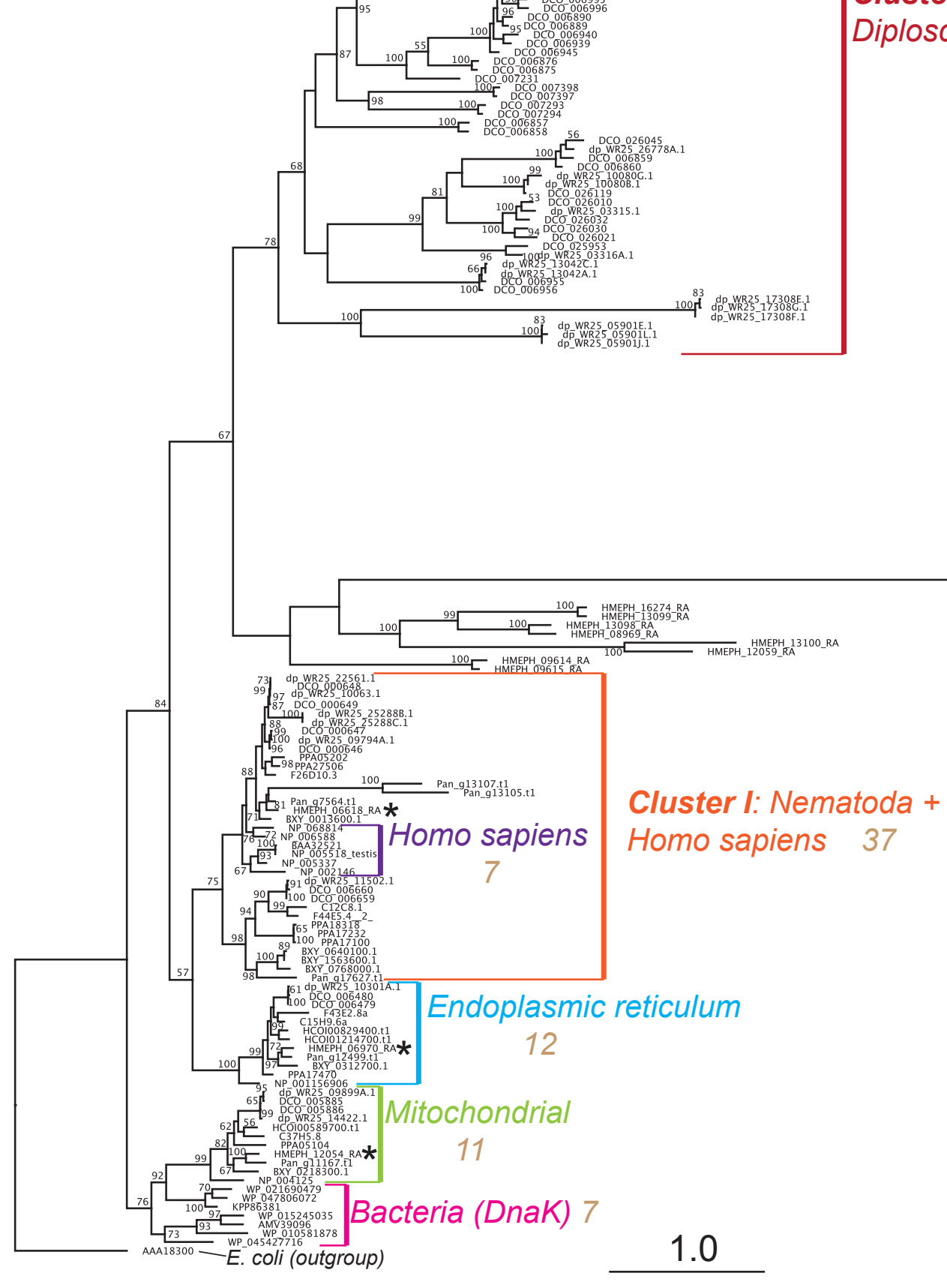




\section{Number of blastp matches to $1,730 \mathrm{H}$. mephisto unknown proteins}

1480

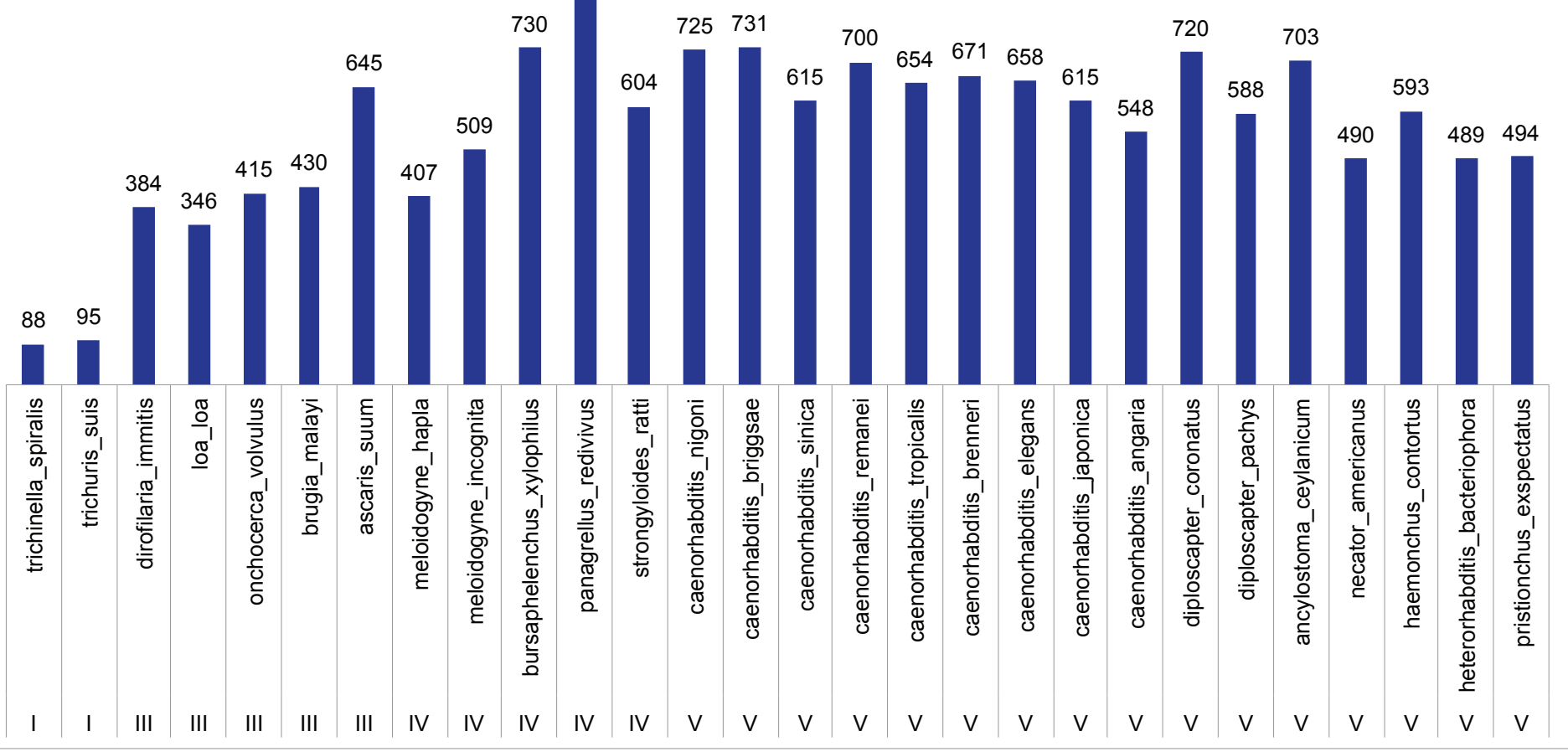

B

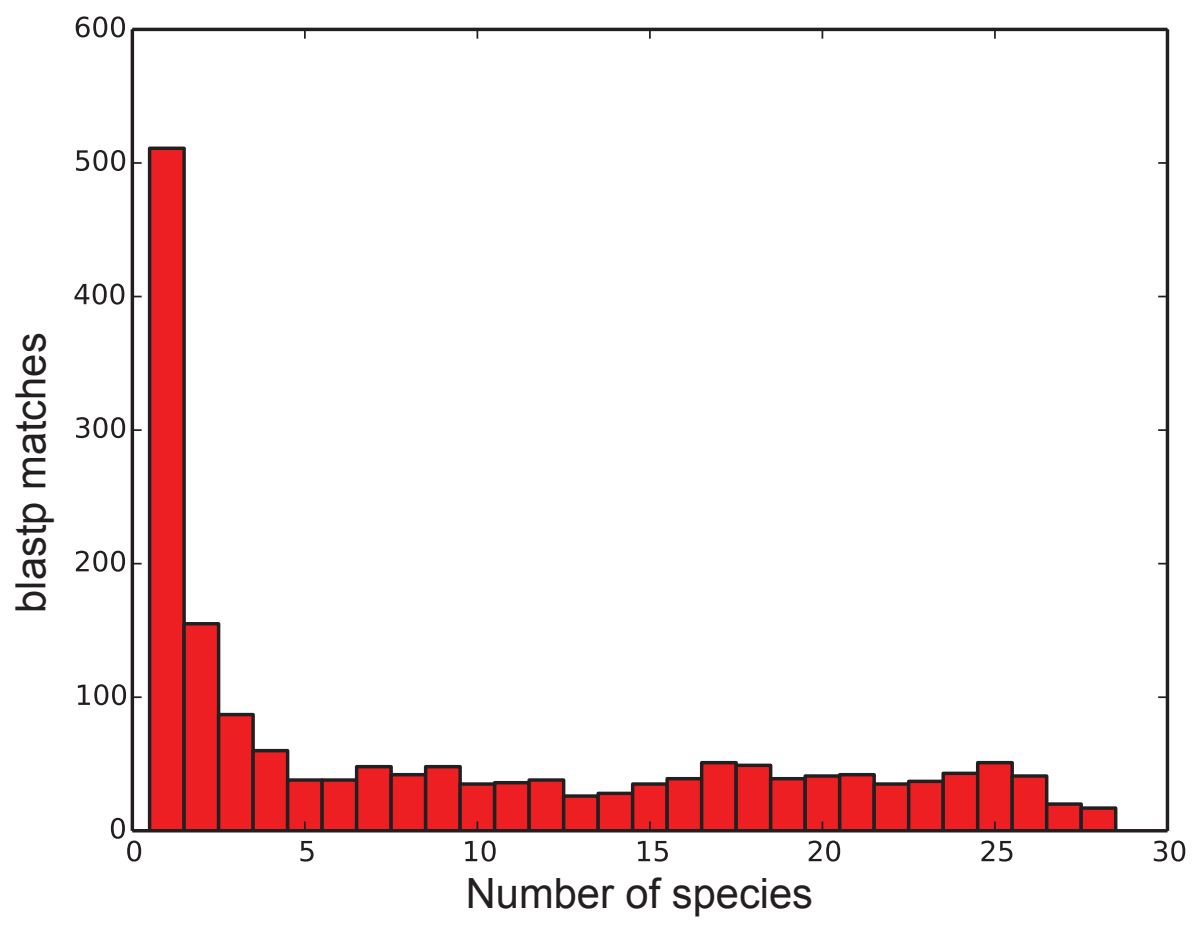




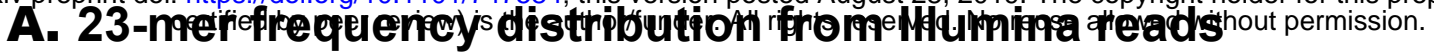

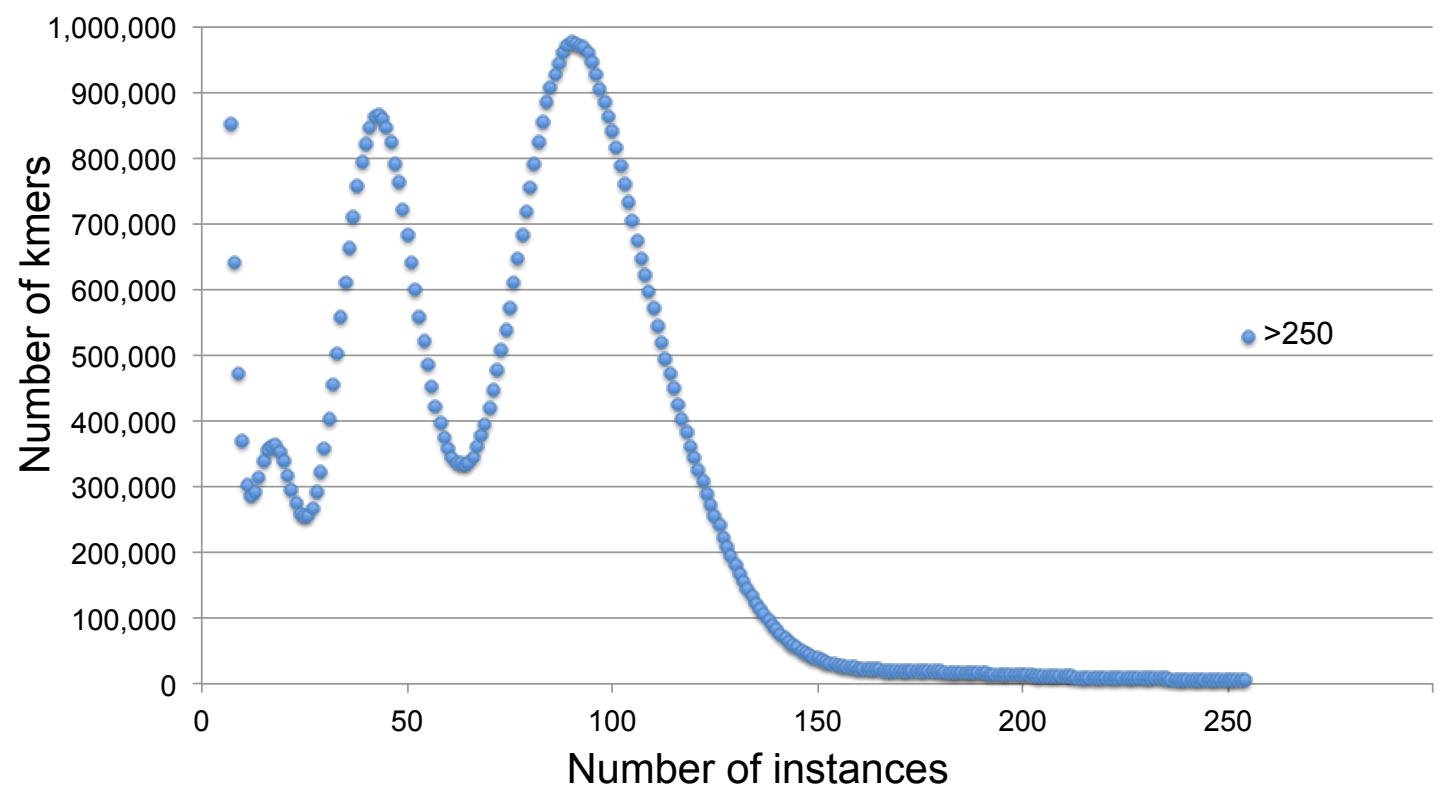

\section{B. Scaffold coverage and \%GC}
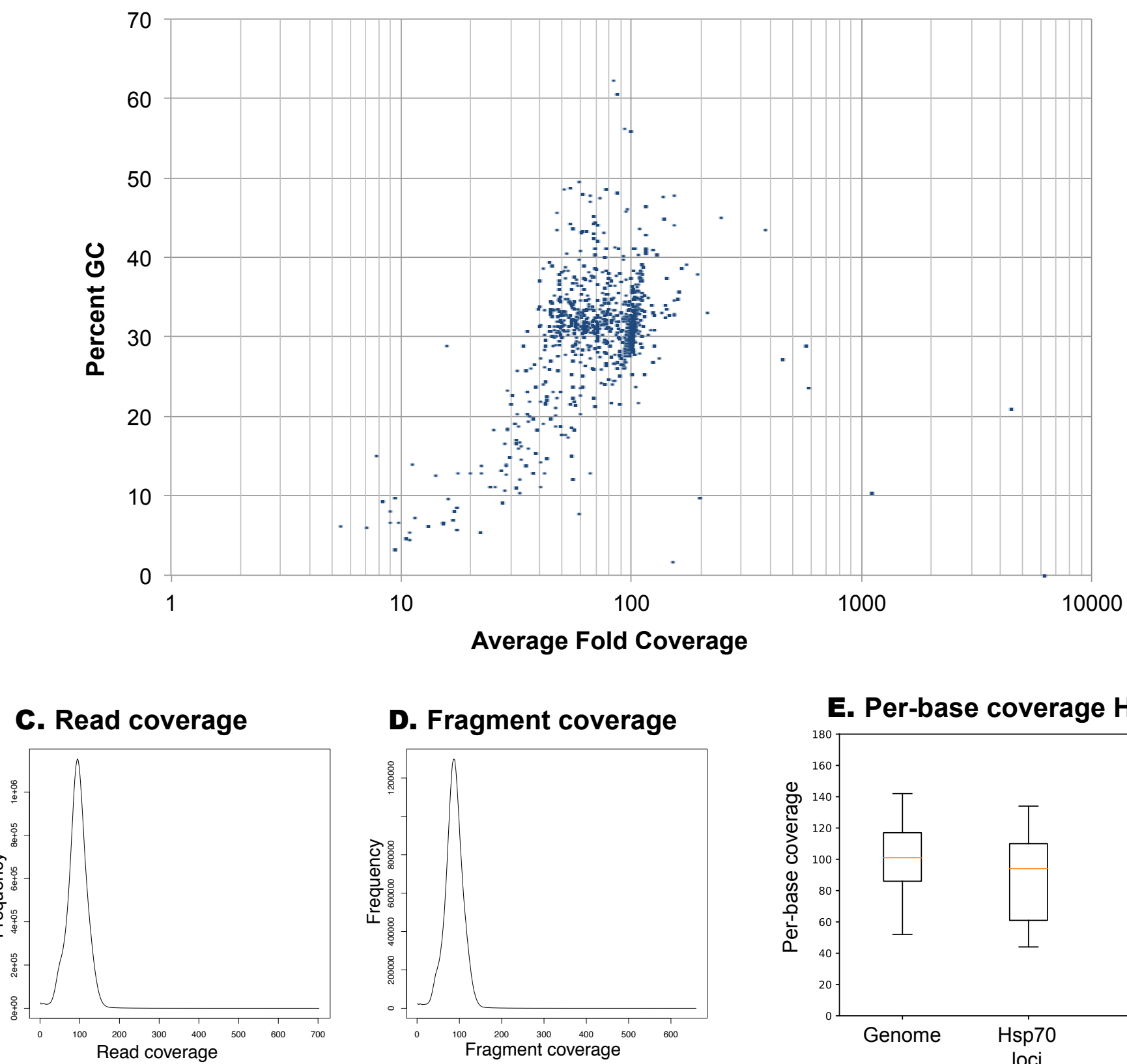

D. Fragment coverage

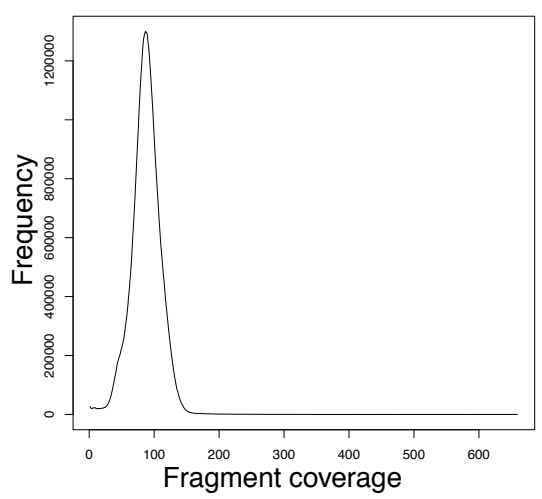

E. Per-base coverage Hsp70, AIG1

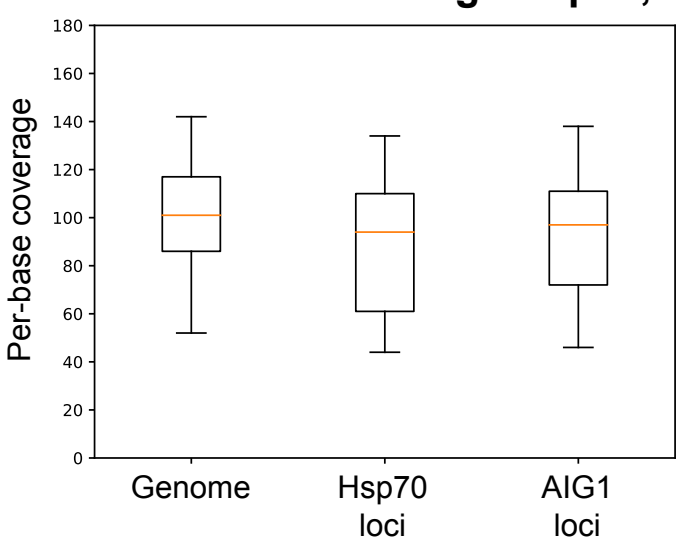


A. Hsp70

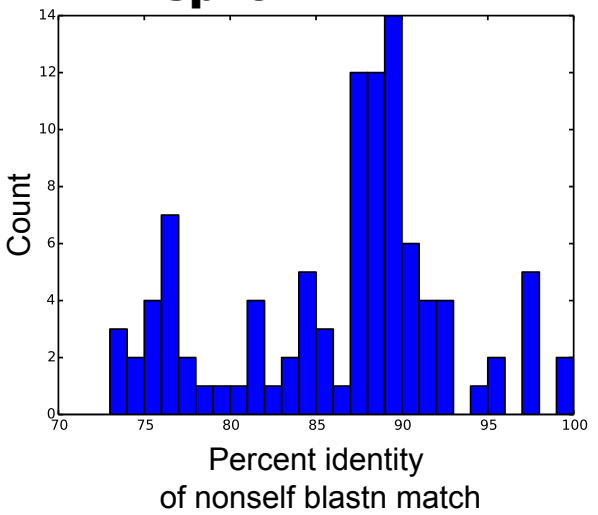

nucleotide sequences with $\mathrm{NO}$ nonself blastn 1e-4 match: $13 / 112$ ( $12 \%$ )

\section{Collagen}

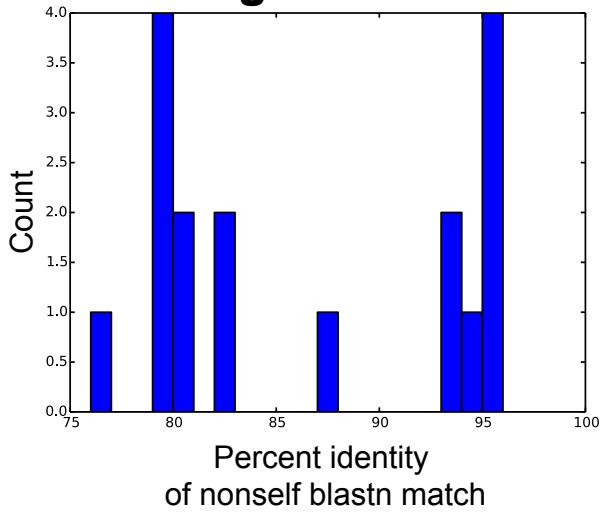

nucleotide sequences with NO nonself blastn 1e-4 match: 48 / 65 ( 74\% )

\section{B. AIG1}

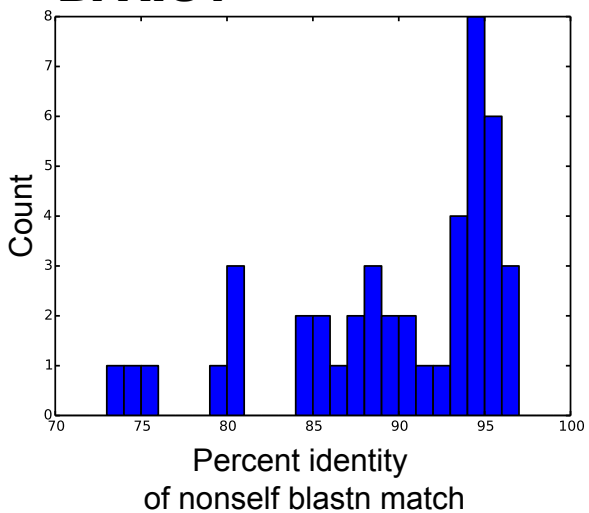

nucleotide sequences with NO nonself blastn 1e-4 match: 19 / 63 (30\% )

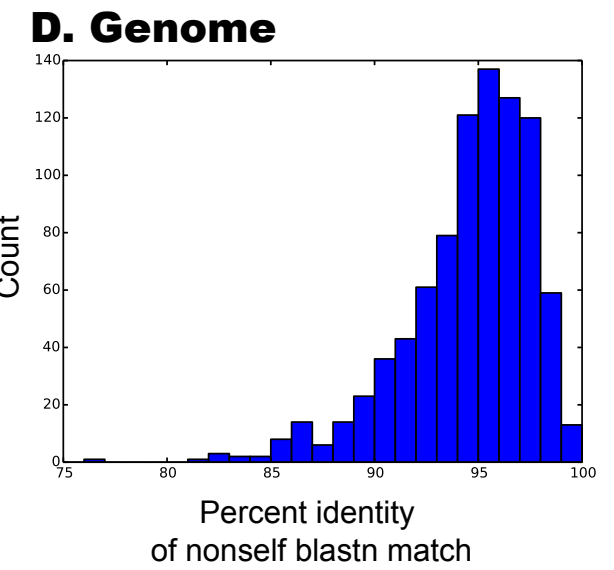

scaffolds with $\mathrm{NO}$ nonself blastn 1e-4 match: 10 / 880 ( 1.1\% ) 